\title{
Observed and simulated depletion layers with southward IMF
}

\author{
N. C. Maynard ${ }^{1}$, W. J. Burke ${ }^{2}$, J. D. Scudder ${ }^{3}$, D. M. Ober ${ }^{1}$, G. L. Siscoe ${ }^{4}$, W. W. White ${ }^{1}$, K. D. Siebert ${ }^{1}$, \\ D. R. Weimer ${ }^{1}$, G. M. Erickson ${ }^{4}$, J. Schoendorf ${ }^{1}$, and M. A. Heinemann ${ }^{2}$ \\ ${ }^{1}$ ATK Mission Research, Nashua, New Hampshire, USA \\ ${ }^{2}$ Air Force Research Laboratory, Hanscom Air Force Base, Massachusetts, USA \\ ${ }^{3}$ Department of Physics and Astronomy, University of Iowa, Iowa City, Iowa, USA \\ ${ }^{4}$ Center for Space Physics, Boston University, Boston, Massachusetts, USA
}

Received: 16 June 2003 - Revised: 21 January 2004 - Accepted: 9 February 2004 - Published: 14 June 2004

\begin{abstract}
We present observations from the Polar satellite that confirm the existence of two types of depletion layers predicted under southward interplanetary magnetic field (IMF) conditions in magnetohydrodynamic simulations. The first depletion type occurs along the stagnation line when IMF $B_{X}$ and/or dipole tilt are/is present. Magnetic merging occurred away from the equator (Maynard et al., 2003) and flux pile-ups developed while the field lines drape to the high-latitude merging sites. This high-shear type of depletion is consistent with the depletion layer model suggested by Zwan and Wolf (1976) for low-shear northward IMF conditions. Expected sites for depletion layers are associated with places where IMF tubes of force first impinge upon the magnetopause. The second depletion type develops poleward of the cusp. Under strongly driven conditions, magnetic fields from Region 1 current closure over the lobes (Siscoe et al., 2002c) cause the high-latitude magnetopause to bulge outward, creating a shoulder above the cusp. These shoulders present the initial obstacle with which the IMF interacts. Flow is impeded, causing local flux pile-ups and lowshear depletion layers to form poleward of the cusps. Merging at the high-shear dayside magnetopause is consequently delayed. In both low- and high-shear cases, we show that the depletion layer structure is part of a slow mode wave standing in front of the magnetopause. As suggested by Southwood and Kivelson (1995), the depletions are rarefactions on the magnetopause side of slow-mode density compressions. While highly sheared magnetic fields are often used as proxies for ongoing local magnetic merging, depletion layers are prohibited at merging locations. Therefore, the existence of a depletion layer is evidence that the location of merging must be remote relative to the observation.
\end{abstract}

Key words. Magnetospheric physics (magnetopause, cusp and boundary layers; magnetosheath; magnetospheric configuration and dynamics)

Correspondence to: N. C. Maynard

(nmaynard@mrcnh.com)

\section{Introduction}

The existence of depletion layers near the magnetopause was first suggested by Midgely and Davis (1963), who reasoned that the magnetic field draping around the magnetopause surface would be constrained to flow in one direction while plasma could flow in all directions. This leads to local magnetic field intensifications and attendant plasma density decreases. Zwan and Wolf (1976) explicitly calculated plasma distributions in the vicinity of the magnetopause to model depletions near the subsolar stagnation point. The model does not allow for magnetic merging (Dungey, 1961) and applies to cases of northward interplanetary magnetic field (IMF). Two mechanisms cause depletion layers. First, deflection by the bow shock around the magnetopause causes plasma to move along the magnetic field lines away from the nose. Second, compressional forces exerted on magnetic flux tubes squeeze plasma away from the subsolar magnetopause along draped field lines. This results in enhanced magnetic fields coupled with decreased plasma density. They predicted depletion factors, ratios of the post-shock to local densities, between 3 and 4. Analyses of IMP-6 measurements showed depletion factors that varied from 1.4 to more than 2 (Crooker et al., 1979).

If merging is occurring at the subsolar magnetopause, plasma flow in the $X$ direction does not completely stagnate, eliminating magnetic field pileup. In a sense, the existence of a depletion layer is an indicator of a locally closed magnetopause, since magnetic merging would carry away the magnetic flux and prevent its buildup. Depletion layers are common near the nose of the magnetopause when the IMF is northward or nearly in the direction of the Earth's dipole magnetic field (low-shear condition). Depletion layers have also been observed for high shear conditions (IMF clock angle greater than $60^{\circ}$ ) (Anderson and Fusilier, 1993). Magnetic merging is commonly believed to be the primary means of coupling the solar wind energy into the magnetosphere. It is important to understand why some high-shear magnetopause crossings have depletion layers, since their existence 
seemingly precludes merging, at least locally. In this paper we explore the causes of high magnetic-shear depletion layers by comparing satellite observations with magnetohydrodynamic (MHD) simulated predictions of the Integrated Space Weather model (ISM) code (White et al., 2001).

AMPTE/IRM data indicate that depletion layers are common at a low-shear $\left(\leq 30^{\circ}\right)$ magnetopause (Phan et al., 1994). With a high-shear $\left(\geq 60^{\circ}\right)$ magnetopause they identified no systematic behavior. Anderson and Fuselier (1993) reported that depletions could be found for all orientations of the IMF but that decreases were small when the IMF had a southward component. Anderson et al. (1997) defined a parameter $\mathrm{D}=E_{R} / E_{S W}$ as an indicator of merging efficiency. Here $E_{R}$ is the reconnection electric field and $E_{S W}$ is the solar wind electric field. $\mathrm{D}=1$ corresponds to no depletion while $\mathrm{D}=0$ corresponds to maximum depletion. They showed that the merging efficiency was a factor of 3 larger during AMTE/IRM (Phan et al., 1994) than AMPTE/CCE (Anderson and Fusilier, 1993) observations. This result was advanced as a reason why Phan et al. (1994) failed to detect systematic depletions during high shear situations. They associated the cause to a 3 -fold higher $\beta$ for the CCE events. Hence, they expect that high-shear depletion layers occur with high solar wind densities. Farrugia et al. (1995) concluded that depletion layers were possible for high magnetic shear whenever the upstream Alfvén mach number was low.

Maynard et al. (2003) used Polar and Cluster data to show that merging often occurs at high latitudes. They described detailed characteristics of an event on 12 March 2001, in which a depletion layer was observed just outside of the magnetopause while merging was occurring poleward of the spacecraft. The event occurred during a high-magnetic-shear interval when the IMF clock angle was $\sim 140^{\circ}$.

Wu (1992) was first to report depletion layers in MHD simulations. The layer was thicker but the depletion factor (1.2) was less than predicted by Zwan and Wolf (1976). More recently, Siscoe et al. (2002a) used ISM simulations to demonstrate the systematic variations of depletion layers with IMF clock angles. Depletion factors $>3$ appear for all cases with the shear $\leq 90^{\circ}$. The thickness of depletion layers decrease with increasing IMF clock angles. Wang et al. (2003) showed from MHD simulations for northward IMF that the depletion layer existed all around the dayside and became thicker away from noon. It was also well defined in the noon-midnight plane past $40^{\circ}$ magnetic latitude. In this paper we wish to emphasize the generality of Zwan-Wolf depletions and related effects at all locations on the magnetopause where the local magnetopause is "passive" (not merging) and particularly at the subset of such locations where the IMF first encounters the magnetopause as a blunt body.

Siscoe et al. (2002a) also pointed out that in ideal, incompressible MHD the stagnation point becomes a line along which the plasma velocity is identically zero. In the absence of merging, the magnetic field that passes through a stagnation point lies on the magnetopause surface and is an MHD mandated stagnation line (Sonnerup, 1974). As such it is a velocity separator line along which the plasma flow diverges.
Motivated by the observation of a density enhancement in front of the magnetopause (Song et al., 1990; 1992), Southwood and Kivelson (1992) argued that a slow MHD shock must form in the magnetosheath behind the bow shock. Depending on pressure anisotropy, it should stand in the sheath flow ahead or behind the intermediate wave (rotational discontinuity) at the magnetopause. A standing slow mode wave would enhance the density and decrease the magnetic field, opposite to the predictions of Zwan and Wolf (1976). Southwood and Kivelson (1995) recognized that the observations of Song et al. $(1990,1992)$ and the mechanism of Zwan and Wolf (1976) both manifest slow mode properties, namely a fluctuation in $\Delta|B|=-\alpha \Delta n$. They suggested that the two models could be reconciled if the slow-mode waves were detached from the magnetopause. Behind the detached shock, magnetic fields should deflect toward the magnetopause. Enhanced magnetic fields and plasma depletions develop at the nose, earthward of the plasma compression at the wave front. The $J \times B$ force of the current in the wave results in a net force that deflects flow away from the nose. We note further in this vain that the wave discussed by Southwood and Kivelson and the depletion predicted by Zwan and Wolf may be viewed as one half cycle of a slow mode disturbance that should be expected in front of any locally closed portion of the magnetopause.

Region 1 current streamlines close through the high latitude boundary layer of the magnetosphere (Siscoe et al., 1991, 2000). These streamlines come out of the dusk side ionosphere, follow magnetic field lines to near the magnetopause in the equatorial region, curl up over the highlatitude magnetopause to the dawn side near equatorial region, and then follow magnetic field lines back to the ionosphere as morning side region 1 currents. A similar loop is located in the Southern Hemisphere. Both loops contribute to the weakening of the magnetic field at the subsolar magnetopause and the increase of it in the lobe. Under strongly driven conditions, the lobes bulge out sunward and the cusps move equatorward (Raeder et al., 2001). Siscoe et al. (2002b) describe the bulge (or deformation of the magnetopause boundary) located above the cusp as a "shoulder", which is the terminology used here. The lobe magnetic field is nearly in the same direction as the draped IMF; hence, this is a locally low-shear boundary encountered above the cusp.

In the following sections we demonstrate that the depletion layer reported in Maynard et al. (2003) occurred near a velocity separator. ISM simulations show that the observations occurred in a region where depletion is expected. We then use ISM simulations to show that under strongly driven conditions with southward IMF, low-magnetic-shear shoulder configurations develop poleward of the cusp. These shoulders impede the flow of magnetic flux toward the nose, requiring the field lines to drape around the shoulder before they can merge at the dayside magnetopause. In so doing they create a depletion layer above the cusp. Polar measurements confirm this ISM prediction. Both types of depletion layers are possible under high-shear, sub-solar magnetopause conditions. The first is tied to merging away from the sub-solar 
region. The second type inhibits dayside merging and is a possible mechanism for understanding the saturation of the ionospheric potential under strongly driven conditions (Siscoe et al., 2002b, c).

\section{Measurements}

In 2001 Polar's apogee $\left(9 R_{E}\right)$ was near the equator. As a consequence, for long intervals in March-April the orbit skimmed from south to north, along the dayside magnetopause. Thus, variations in phenomena observed by Polar are primarily temporal rather than spatial. Information about radial scale sizes is lost. However, Polar closely monitors magnetopause responses to temporal changes of the IMF and solar-wind pressure. Several sensors on Polar are used in this study. The Hydra Duo Deca Ion Electron Spectrometer (DDIES) (Scudder et al., 1995) consists of six pairs of electrostatic analyzers looking in different directions to acquire high-resolution energy spectra and pitch-angle information. Full three-dimensional distributions of electrons with energies between $1 \mathrm{eV}$ and $10 \mathrm{keV}$ and ions with energies per charge ratio of $10 \mathrm{eV} \mathrm{q}^{-1}$ to $10 \mathrm{keV} \mathrm{q}^{-1}$ were sampled every $13.8 \mathrm{~s}$. The electric field instrument (EFI) (Harvey et al., 1995) uses a biased double probe technique to measure vector electric fields from potential differences between 3 orthogonal pairs of spherical sensors. This paper presents measurements from the long wire antennas in the satellite's spin plane. The Magnetic Field Experiment (MFE) (Russell et al., 1995) consists of two orthogonal tri-axial fluxgate magnetometers mounted on non-conducting booms. The electric and magnetic fields were sampled 40 and $8 \mathrm{~s}^{-1}$, respectively. Data presented in this paper were spin averaged using a leastsquares fits to a sine function.

The Advanced Composition Explorer (ACE) spacecraft monitors interplanetary conditions while flying in a halo orbit around the $L_{1}$ point in front of the Earth. The solar wind velocity was measured by the Solar Wind Electron, Proton, and Alpha Monitor (SWEPAM) (McComas et al., 1998). A tri-axial fluxgate magnetometer measured the interplanetary magnetic field vector (Smith et al., 1998).

\section{The Integrated Space Weather Prediction Model}

The Integrated Space Weather Prediction Model (ISM) operates within a cylindrical computational domain whose origin is at the center of the Earth. Its domain extends $40 R_{E}$ sunward, and $300 R_{E}$ in the anti-sunward direction, and $60 R_{E}$ radially from the Earth-Sun line. In simulations described here, the cylindrical domain has an interior spherical boundary approximately located at the bottom of the E-layer $(100 \mathrm{~km})$. The cylindrical to spherical interface is at $3 R_{E}$.

ISM uses standard MHD equations augmented by hydrodynamic equations in the collisionally-coupled thermosphere. Conceptually, as one moves toward the Earth, equations transition continuously from pure MHD in the solar wind and magnetosphere to those proper to the low-altitude ionosphere/thermosphere. The simulations discussed here contain specifically selected parameters and simplifying approximations. Finite-difference grid resolution varies from a few hundred kilometers in the ionosphere to several $R_{E}$ near the computational domain's outer boundary. At the magnetopause, resolution ranges from 0.2 to $0.8 R_{E}$. Explicit viscosity in the plasma momentum equation was set to zero. To approximate nonlinear magnetic reconnection the explicit resistivity coefficient $v$ in Ohm's Law equation is zero if current density normal to $B$ is less than $3.16 \cdot 10^{-3} \mathrm{Am}^{-2}$. In regions with perpendicular current densities above this threshold, $v=2 \cdot 10^{10} \mathrm{~m}^{2} \mathrm{~s}^{-1}$. In practice, this choice of $v$ leads to non-zero explicit resistivity near the subsolar magnetopause, and in the nightside plasma sheet. Dissipation, where needed to maintain numerical stability, is based on the partial donorcell method (PDM) as formulated by Hain (1987).

In the following discussion we present the results of five simulations. The input solar wind parameters for each run are given in Table 1. Because of their negligible impact on the results and in order to reduce run times of simulations, routines in the ISM code containing thermospheric hydrodynamics and explicit chemistry between ionospheric and thermospheric species were not activated. Ionospheric Pedersen conductance is $6 \mathrm{~S}$ at the pole and varied in latitude as $B^{-2}$. It is uniform in longitude. No Hall conductance was used.

\section{Depletion layers with northward IMF}

To set the stage for the depletion layers when the IMF has southward components, we first examine conditions on 31 March 2001, during a period when the IMF was strongly northward and Polar's orbit was skimming along the dayside magnetopause near the equator. Data from 08:00 to 11:00 UT are plotted in Fig 1. Figures 1d-g present energy-versus-time spectrograms of ion energy fluxes measured by HYDRA at all pitch angles and parallel, perpendicular, and antiparallel to the local magnetic field. The plotted numbers are the average of $d^{2} f / d E / d \Omega$ over solid angles in which data were taken. Some investigators integrate over solid angle and report $\int\left(d^{2} f / d E d \Omega\right) d \Omega$ as the "omnidirectional" energy flux. The entire velocity distribution at all solid angles must be sampled to make such a determination. We avoid interpolating and report the average of the integrand over all directions sampled, which at a $13.8 \mathrm{~s}$ cadence covers $12 * 6=72$ directions distributed over a unit sphere. Figure 1a, the black trace, shows $B_{Z}$ measured by Polar. The green trace indicates $B_{Y}$. In Fig. $1 \mathrm{~b}$ the blue line indicates the solar wind density measured by ACE and lagged by $38 \mathrm{~min}$. While the lag time may vary minute-by-minute (Weimer et al., 2002), we have chosen $38 \mathrm{~min}$ as providing the best average match over the plotted interval. In this case the lag time is close to the advection time and is adjusted by matching clock angles of magnetic fields measured by Polar and ACE (Song et al., 1992). Matching clock angles provides reasonable lags for both $B$ and density, except when IMF $B_{X}$ is the dominant component. Figure 1c compares the magnitude of the 
Table 1. Simulation input parameters.

\begin{tabular}{ccccccc}
\hline$\#$ & $\begin{array}{l}\text { Solar Wind velocity } \\
\text { velocity }(\mathrm{km} / \mathrm{s})\end{array}$ & $\left.\begin{array}{l}\text { Solar wind density } \\
\text { density (protons/cm }\end{array}{ }^{3}\right)$ & $\begin{array}{l}\text { IMF magnitude } \\
(\mathrm{nT})\end{array}$ & $\begin{array}{l}\text { IMF clock } \\
\text { angle (degrees) }\end{array}$ & IMF $B_{X}$ & $\begin{array}{l}\text { Dipole } \\
\text { tilt (degrees) }\end{array}$ \\
\hline 1 & 350 & 5 & 5 & 135 & negative & 0 \\
2 & 350 & 5 & 5 & 180 & 0 & 17 \\
3 & 350 & 5 & 20 & 180 & 0 & 17 \\
4 & 350 & 5 & 20 & 180 & 0 & 35 \\
5 & 350 & 5 & 50 & 180 & 0 & 0 \\
\hline
\end{tabular}

\section{3/31/01: 0900 UT: Bz north depletion layer at nose}

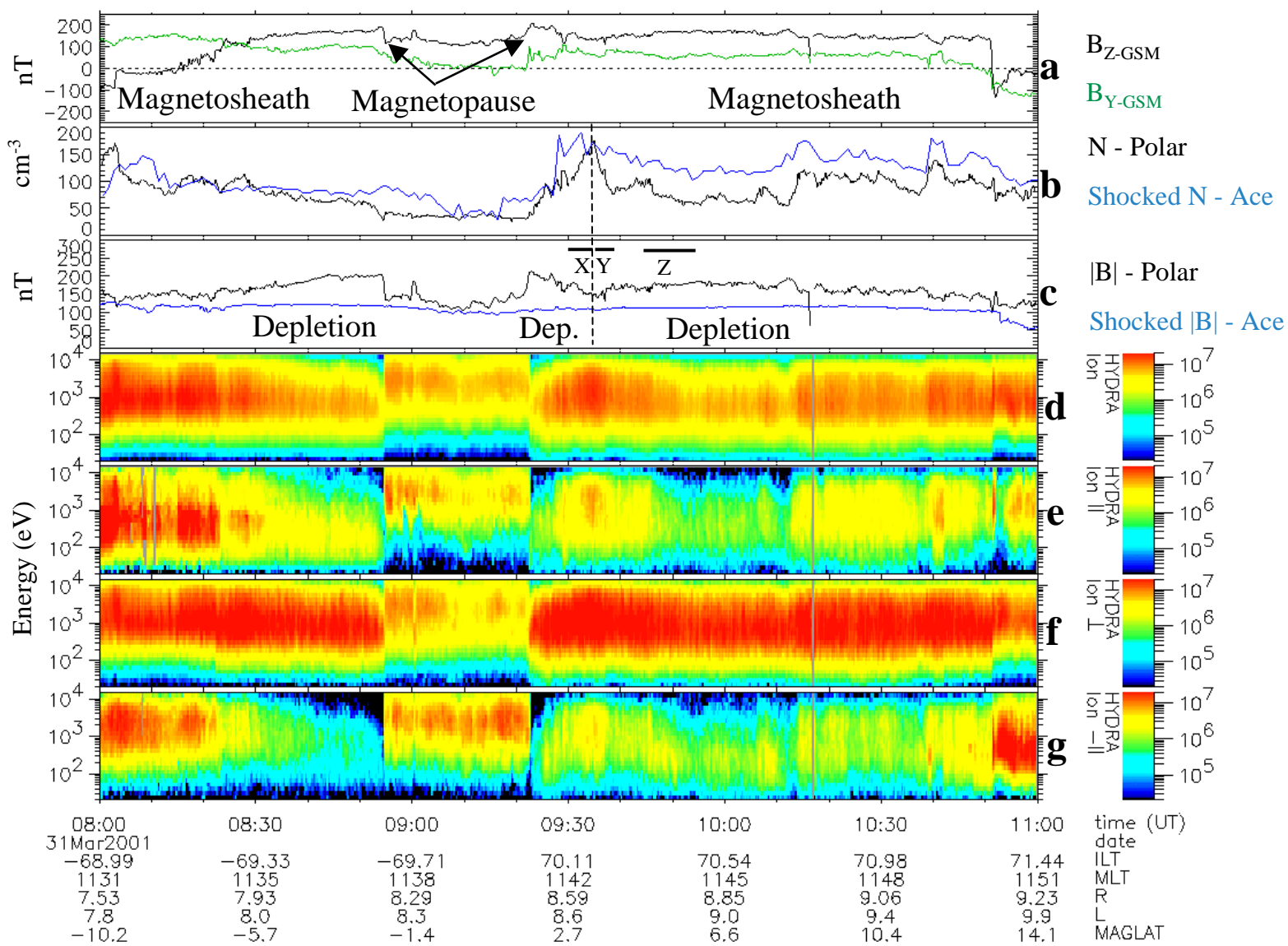

Polar at nose near $0^{\circ}$ MLAT and 1140 MLT

Fig. 1. Example from Polar of depletions layers with IMF $B_{Z}$ northward. Polar was located at $0^{\circ}$ MLAT and 11:40 MLT. (a) The black trace is the measured $B_{Z}$ at Polar. The green trace is the measured $B_{Y}$. The larger value of $B_{Z}$ just outside the magnetopause is a signature of a depletion layer. (b) The measured ion density at Polar (black) compared to the properly lagged (38 min) ion density measured at ACE upstream in the solar wind (blue). Plasma densities attributed to ACE have been multiplied by a time-varying factor appropriate for density jumps predicted by the Rankine-Hugoniot relations for the subsolar bow shock and by an additional compression factor when Polar was close to the magnetopause as predicted by Spreiter and Stahara (1985). (c) The magnetic field magnitude measured at Polar (black) and at ACE (blue). ACE magnetic field measurements were multiplied by the time-varying Rankine-Hugoniot factor appropriate for the magnetic field jumps at the subsolar bow shock. (d-g) Ion energy spectrograms showing the total, parallel (pitch angles $\left.0-30^{\circ}\right)$, perpendicular $(75-$ $\left.105^{\circ}\right)$ and antiparallel $\left(150-180^{\circ}\right)$ ion fluxes $\left(\left(\mathrm{cm}^{2}-s-s r-\Delta E / E\right)^{-1}\right)$. Three intervals noted by $\mathrm{X}, \mathrm{Y}$, and $\mathrm{Z}$ indicate the data on which the regression analyses of Fig. 11 were performed. 


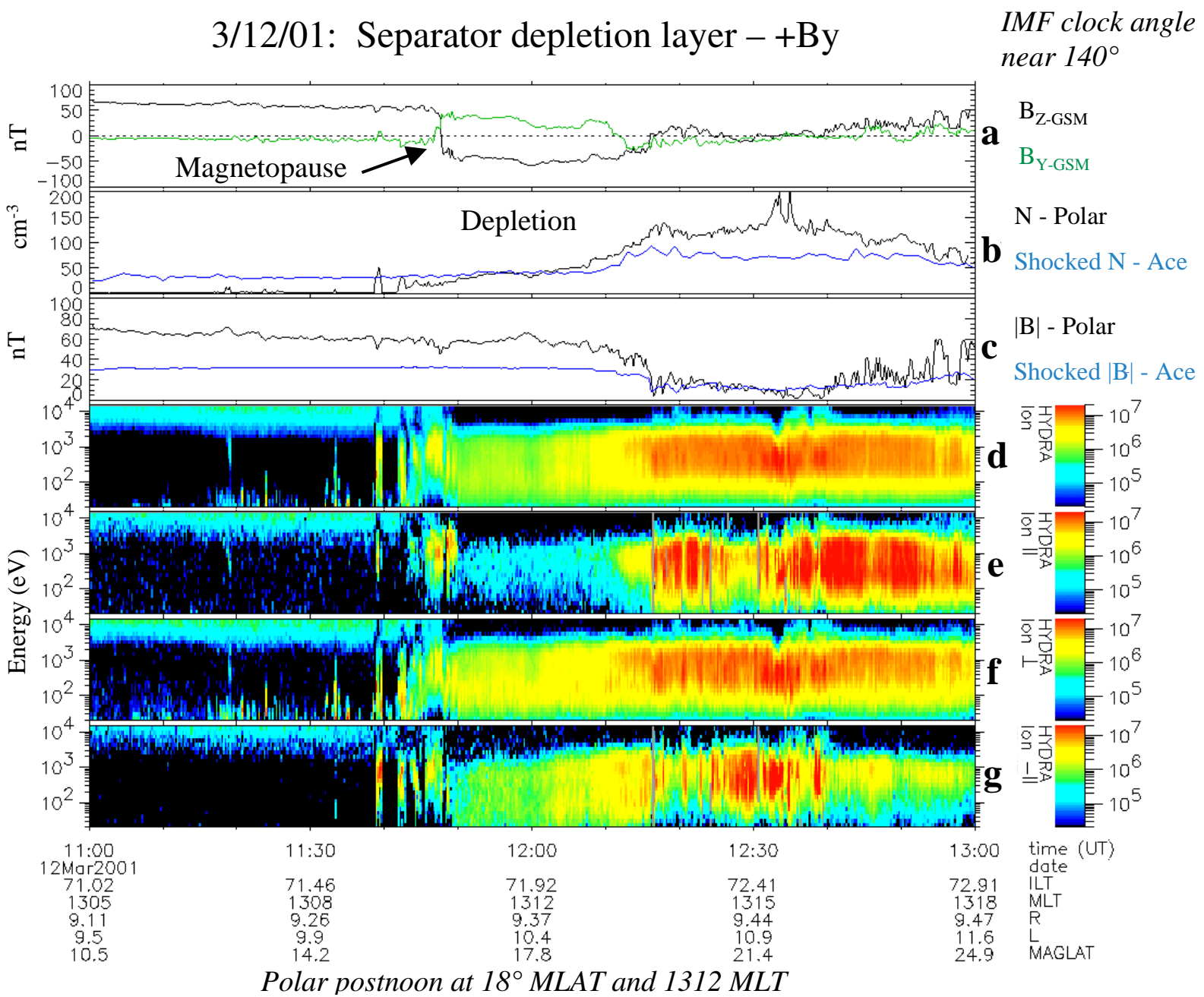

Fig. 2. Example of a depletion layer for negative $B_{Z}$ and positive $B_{Y}$ in the same format as Fig. 1 . Polar was located at $18^{\circ}$ MLAT and 13:12 MLT.

magnetic fields measured by Polar and ACE with the same time delay. To make the comparisons with Polar data, it is necessary to adjust the ACE density and magnetic field for effects of the bow shock. The ACE magnetic field and solar wind density have been multiplied by time varying factors calculated using the reduction of the Rankine-Hugoniot equations by Whang (1987), which predicts the jump factors based solely on the upstream conditions. As such, the values represent the expected magnetosheath density and magnetic field just inside the bow shock. All factors were calculated for the conditions at the nose. Typical factors for density range between 3 and 4 . The magnetic field factor is more variable, depending on whether the shock is more perpendicular or parallel. They range from 1 for a purely parallel to the factor appropriate for the density with a perpendicular shock. ACE density measurements were adjusted to allow for latitudinal variations of the compressed magnetosheath plasma, as prescribed by Spreiter and Stahara (1985). No further adjustment was made on the ACE magnetic field since their compression depends on several factors, negating possible improvements with a simple change. Hydra densities were calibrated against those measured by ACE during the interval between 12:30 and 13:00 UT when Polar was in the solar wind.

At the location of Polar the imposed IMF turned northward near 08:20 UT and the magnetosheath ion distributions became increasingly dominated by the perpendicular fluxes, or "pancake" shaped, as antiparallel and parallel fluxes disappeared. The magnetic field's magnitude increased by a factor of 1.7 above the shocked IMF (Fig. 1c). The density decreased (Fig. 1b) relative to the adjusted solar wind density. The lower density and increased magnetic field just outside the magnetopause are the predicted signatures of depletion layers (Zwan and Wolf, 1976). The pancake ion distributions are a natural result of the Zwan and Wolf mechanisms. This trend continued until 08:54 UT when the spacecraft crossed the magnetopause into the boundary layer, marked by an abrupt change in ion spectral characteristics and a decrease in $B$ and $B_{Z}$. During magnetopause crossings with no depletion layers, the magnetic field is expected to be larger in the 


\section{Particle source compared to dawn-dusk velocity $\sim 140^{\circ}$ clock angle}

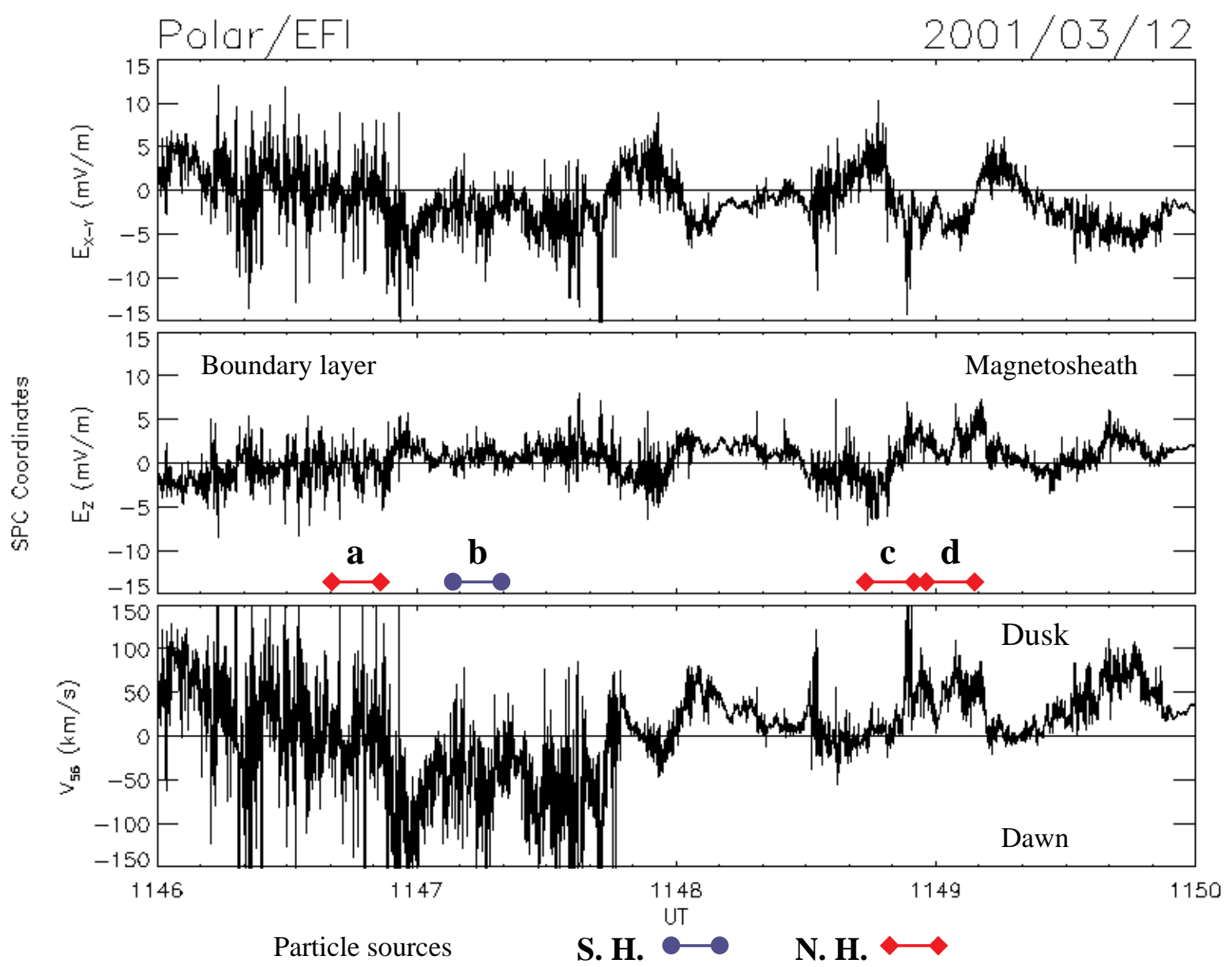

Fig. 3. High resolution $(40 \mathrm{~Hz})$ measurements of the 2 components of the electric field in the spin plane and the resulting drift velocity along the spin axis, which is roughly in the $Y$ direction (toward dusk). The letters refer to panels in Fig. 8 of Maynard et al. (2003). The circular dumbells represent intervals when fluxes are coming from below the satellite, while the diamond dumbells represent times when ions are coming from a Northern Hemisphere source above the spacecraft.

lower density boundary layer. Polar crossed back into the depletion layer at 09:22 UT as the increased density observed by ACE (blue trace in Fig. 1b) rapidly pushed the magnetopause inward. The depletion layer was quickly crossed, and at 09:35 UT there was a significant increase in density and a decrease in magnetic field. We return to this point in Sect. 7. After 09:40 UT the plasma density at ACE decreased and the magnetopause expanded to closer to the location of Polar, placing the satellite back into the more depressed density of the depletion layer, where it remained for a considerable interval. Compared with shocked ACE measurements, the maximum increase in $B$ at Polar was by a factor of 2 . Note that at both ends of Fig. 1c the magnetic field magnitude returns to close to the shocked IMF value, while in Fig. 1a the $Z$ component is southward, favorable to dayside merging with no depletion. Hence, variations in measurements at Polar reflect temporal responses to boundary motions and the solar wind drivers. However, we cannot use Polar measurements to determine the thickness of the depletion layer.
The examples with southward IMF components that follow show data in the same format as Fig. 1. Evidence for depletion layers include (1) ion distributions favoring perpendicular pitch angles, (2) densities at Polar below the shocked ACE values, and (3) increased magnetic fields above the shocked ACE values. Ion distributions in the sub-solar magnetosheath become more pancake shaped as the magnetic flux piles up in the stagnated region.

\section{Velocity separator depletion layers}

Maynard et al. (2003) identified a merging event at high latitudes during the Polar magnetopause crossing at 11:48 UT on 12 March 2001. Accelerated ions and Poynting flux carried by Alfvén waves were observed coming from poleward of the spacecraft, on both the magnetosphere and magnetosheath sides of the current layer. After crossing the magnetopause Polar entered a region of low plasma density within the magnetosheath. Figure 2 presents HYDRA and MFE 
data from Polar for this crossing (some of which is repeated from Fig. 6 of Maynard et al., 2003). Figure 2 is presented in the same format as Fig. 1. Figures $2 \mathrm{~d}-\mathrm{g}$ show ion energy fluxes in spectrogram formats. Figure 2 a presents IMF $B_{Y}$ and $B_{Z}$ components measured by Polar as being comparable with an average clock angle of $\sim 140^{\circ}$. A positive IMF $B_{X}$ also was present. Note that just outside the magnetopause crossing the parallel and anti-parallel ion fluxes were very small. Perpendicular fluxes dominated but at reduced levels compared to those detected after 12:15 UT. Figure $2 \mathrm{~b}$ compares the density measured by Polar with that measured by the ACE satellite. Here ACE data were lagged by $63 \mathrm{~min}$, which proved optimal for the magnetopause crossing as determined in Maynard et al. (2003). Ion densities detected just outside the magnetopause were less than or comparable to those of the shocked solar wind as observed by ACE. B remained above the shocked IMF value until a major IMF decrease occurred near 12:18 UT. The maximum enhancement of the magnetic field was by a factor of 1.9. Reduced densities and corresponding enhancements in $B$ are clear indications of depletion layers. Note that the density minimum observed at 12:00 UT does not correspond to a change in the solar wind density, but to an increase in the total magnetic field (Fig. 2c). Temporal changes in the depletion, such as observed at 12:00 UT, most likely reflect a small movement of the magnetopause, placing the satellite deeper inside the layer.

Signatures of accelerated ions appear in three of the four particle distributions shown in Fig. 8 of Maynard et al. (2003), to demonstrate that merging was occurring at high latitudes to the north of the spacecraft. The accelerated ions in the other example came from the south. This suggests that Polar was located near the velocity separator line, or the magnetic field line that passes through the stagnation point at the nose. Sonnerup (1974) showed that in incompressible MHD the stagnation point stretches into a line along $B$. Later, Siscoe et al. (2002a) demonstrated this effect with the ISM code. On the dawn (dusk) side of this line plasma flows toward dawn (dusk). To test the conjecture that Polar was in the vicinity of the stagnation line, Fig. 3 plots the two electric field components measured in the spin plane of the spacecraft and the velocity along the spin axis calculated using those components during the magnetopause crossing. The spin axis points close to the dawn-dusk direction. The intervals over which the particle distributions shown in Fig. 8 of Maynard et al. (2003) were taken are noted with square (circular) dumbbells for distributions originating north (south) of the spacecraft. The letters refer to the labels in Fig. 8 of Maynard et al. (2003). Where the particle source was south (north) of the spacecraft or from the Southern (Northern) Hemisphere, the velocity was toward dawn (dusk), as anticipated. This is consistent with Polar being located near the stagnation line or velocity separator.

Since incoming magnetic flux in the magnetosheath drapes over the magnetopause before reaching high-latitude merging sites, flux pile up must occur near this stagnation line and a depletion layer should form (Midgely and Davis, 1963;
Zwan and Wolf, 1976). Positive IMF $B_{X}$ causes flux to encounter the stagnation line north of the equator first, enhancing the flux pile up at that point. Note that if component merging had been occurring near the equatorial region, no flux pile up would have developed as field lines would have been immediately dragged in the dawn or dusk direction.

To explore this scenario, we utilized an existing ISM simulation (Simulation 1 in Table 1) with equal magnitudes for all IMF components; $B_{X}$ and $B_{Z}$ negative and $B_{Y}$ positive. The negative IMF $B_{X}$ places the first contact point to the stagnation line south of the equator on the dawn side of noon, comparable to the observations at Polar on the dusk side of noon north of the equator with IMF $B_{X}>0$. Figure 4 a shows ion velocity vectors and contours of $B_{Z}$ in the $Y Z$ plane at $X=9.9 R_{E}$. The tan colored region represents the nose of the magnetosphere with positive $B_{Z}$; the color blue indicates negative $B_{Z}$ and open field lines. In the region of open field lines noted by arrows, Figs. $4 \mathrm{~b}$ and $\mathrm{c}$ show decreased density and increased magnetic field, respectively. The simulation provides the signatures of a depletion layer in the region of the first contact with the stagnation line, comparable to the Polar measurements described above. The depletion in density of about 1.3 is less than measured but comparable to values of 1.3 and 1.2 reported by Wu (1992) and Lyon (1994), respectively, in depletion-layers simulations with northward IMF, using other MHD codes. The depletion is localized south of the equator and primarily in the vicinity of the stagnation line, although it also extends across noon with somewhat weaker levels of enhanced $B$. The negative $B_{X}$ in the simulation places the first contact point south of the equator, in the prenoon sector (Fig. 4). Had the simulation run with positive IMF $B_{X}$, the first contact point and strongest signature of depletion would occur north of the equator in the postnoon sector. This compares favorably with the location of Polar. The presence of IMF $B_{X}$ can enhance the depletion as does dipole tilt. Maynard et al. (2003) showed that the merging site moved off the equator and away from the point of first contact with increasing dipole tilt. IMF $B_{X}>0$ at Polar serves to enhance the effective dipole tilt and increase the depletion. Siscoe et al. (2002a) showed that the depletion is minimal when IMF $B_{X}=0$ and the dipole axis is vertical. The simulation has a current-dependent, explicit resistivity at the nose, which favors merging at that location. In the simulation the neutral line moved away from the subsolar region, indicating a strong preference for high-latitude merging when the dipole is tilted.

If IMF $B_{Y}$ had been negative and $B_{X}$ positive, the depletion layer would have been located on the dawn side of noon in the Northern Hemisphere. Such a case was observed briefly at $\sim 11: 30$ MLT (near 08:00 UT) on 16 April 2000, when Polar was moving northward and toward dusk along the magnetopause. Figure 5 displays similar quantities to those in Figs. 1 and 2 between 06:00 and 11:00 UT. Polar initially crossed the magnetopause at 07:10 UT. Approaching the current layer on the magnetosphere side, Fig. 5a shows that the angle of $\mathrm{B}$ rotates to $\sim 45^{\circ}$ as the current layer is entered and then to $90^{\circ}$ IMF orientation on the outside of the 

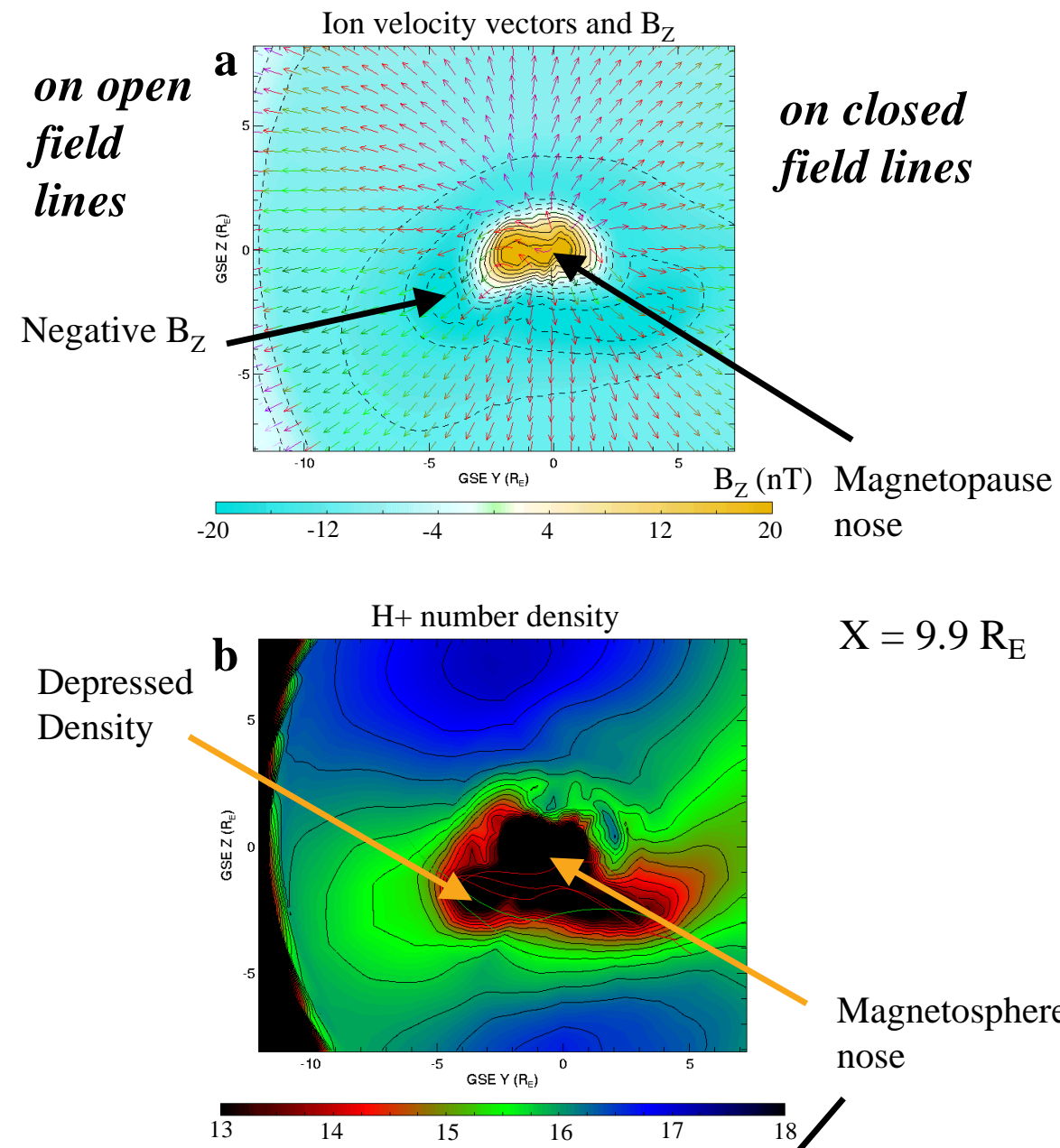

Magnetosphere nose

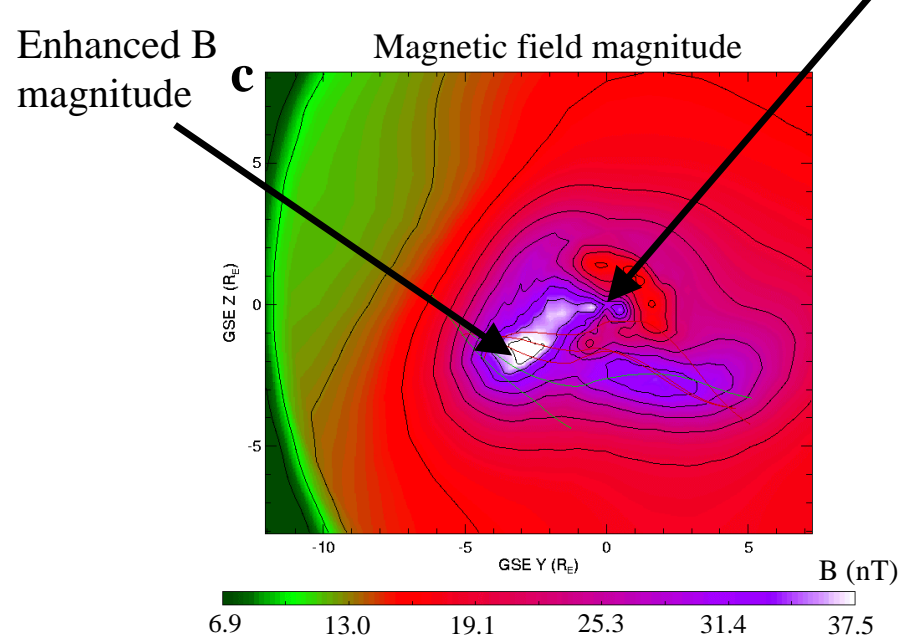

IMF: $-\mathrm{B}_{\mathrm{X}} ;+\mathrm{B}_{\mathrm{Y}} ;-\mathrm{B}_{\mathrm{Z}}$

Fig. 4. Results from a MHD simulation with a constant IMF input of equal magnitude negative $B_{X}$, positive $B_{Y}$ and negative $B_{Z}$. The simulation output is presented in the $Y Z$ plane at $X=9.9 R_{E}$, which just contacts the nose of the magnetosphere. (a) Velocity vectors are overlaid on the plane colored with $B_{Z}$. Brown colors indicate closed magnetosphere field lines, while blue colors indicate open magnetosheath or boundary layer field lines. (b) The plane is colored with the proton number density. (c) The plane is colored with the magnitude of the magnetic field. Attention is called to the region below and to the left of center in each panel, where depressed density and enhanced $B$ are seen on open field lines. 


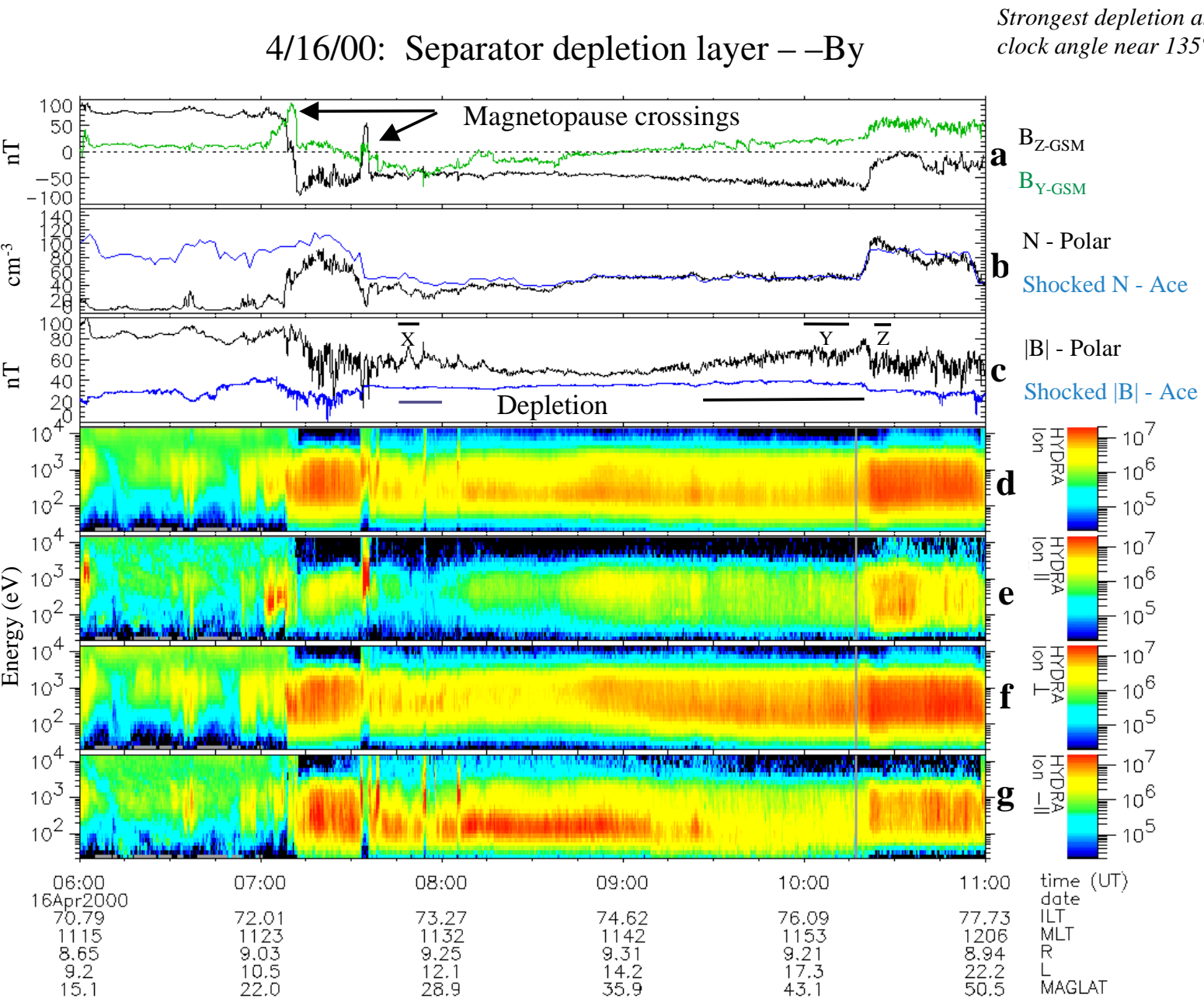

Fig. 5. An example from Polar located prenoon at $30^{\circ}$ MLAT and 11:32 MLT in the same format as Fig. 1. The signatures of depletion are seen briefly just before 08:00 UT when IMF $B_{Y}$ is negative and comparable to $B_{Z}$. Subsequently, as the magnitude of $B_{Y}$ decreases, antiparallel ion fluxes and the total ion density return from merging somewhere below the spacecraft. Depletion is seen on the dusk side of noon for the opposite polarity of $B_{Y}$ near 10:00 UT. Three intervals noted by X, Y, and Z indicate the data on which the regression analyses in Fig. 12 were done.

current layer. This rotation in the boundary layer is consistent with MHD simulations (Maynard et al., 2001). The IMF and Polar B quickly rotated from a clock angle of $90^{\circ}$ to a strongly negative $B_{Z}$, concurrent with an increase in the density. At this prenoon local time and $22^{\circ}$ magnetic latitude, the magnetosheath density followed the adjusted solar wind density, but at a slightly lower level. The magnitude of $B$ increased. However, the ion fluxes were strongest in the direction parallel to the magnetic field. The ion temperature was higher than that observed in the magnetosheath after the next magnetopause crossing. The solar wind density decreased near 07:30 UT, and the magnetopause briefly expanded to the location of Polar at 07:35 UT. Beyond this second outbound magnetopause crossing $B_{Y}$ became negative and comparable in magnitude to $B_{Z}$. At this time the velocity separator location would be prenoon in the vicinity of the satellite. Near 08:00 UT evidence of a mild depletion layer is seen in the density and magnetic field (noted by the blue bar). The de- pletion encounter was brief, becoming most intense after a flux transfer event at $\sim 07: 54 \mathrm{UT}$. Soon after this $B_{Y}$ returned to zero, and the depletion interval ended as ion densities measured by Polar increased to the adjusted ACE densities.

A second region of depletion, identified by enhanced $B$ and pancake particle distributions, appears in Fig. 5 after 09:30 UT. Polar was located near magnetic noon and at a magnetic latitude $>40^{\circ}$. $B_{Y}$ was positive, similar to the conditions of 12 March 2001. Figure 4 shows that the region of depressed density and enhanced $B$ extends to noon from the maximum near the velocity separator, suggesting that Polar accessed the depletion region. The depletion increased with time as evidenced by the increase in $B$ and the decrease in parallel and antiparallel ion fluxes until 10:10 UT. The density remained relatively constant near adjusted ACE values; however, the electron pressure and temperature (not shown) decreased commensurate with the increase in $B$. Near 10:20 UT an increase in the solar wind density pushed 


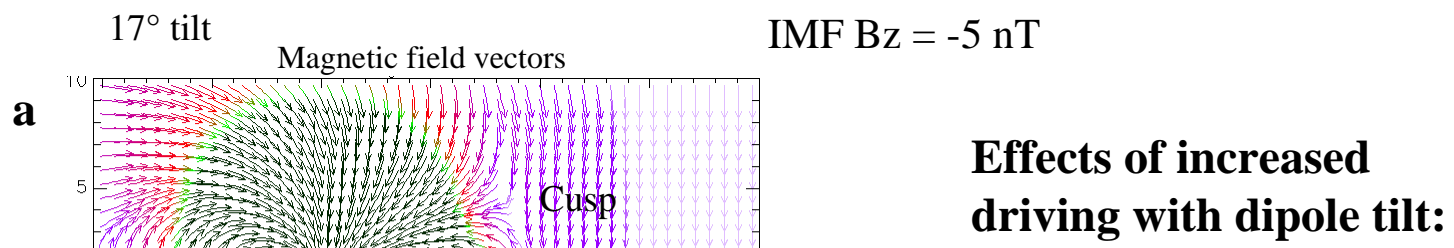

Narrows distance
between cusps

Creates a shoulder above cusps

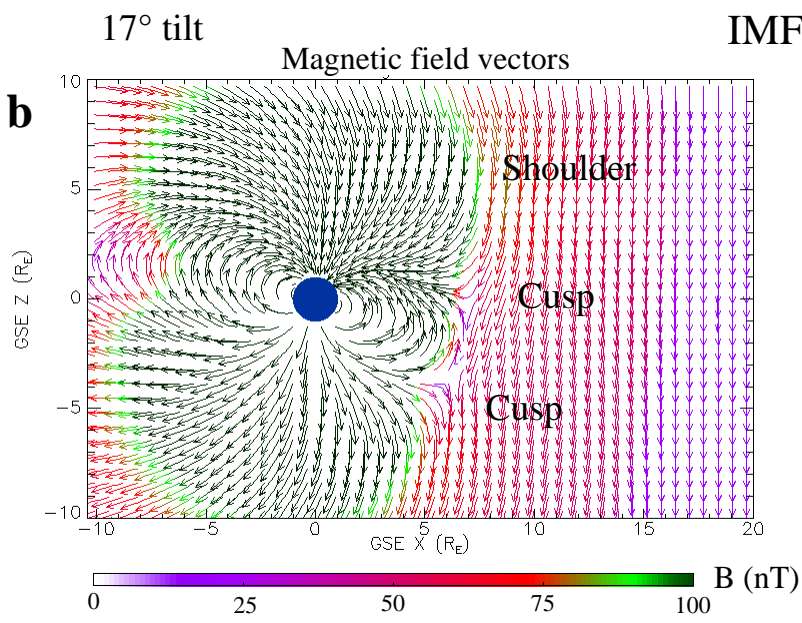

$\mathrm{MF} \mathrm{Bz}=-20 \mathrm{nT}$

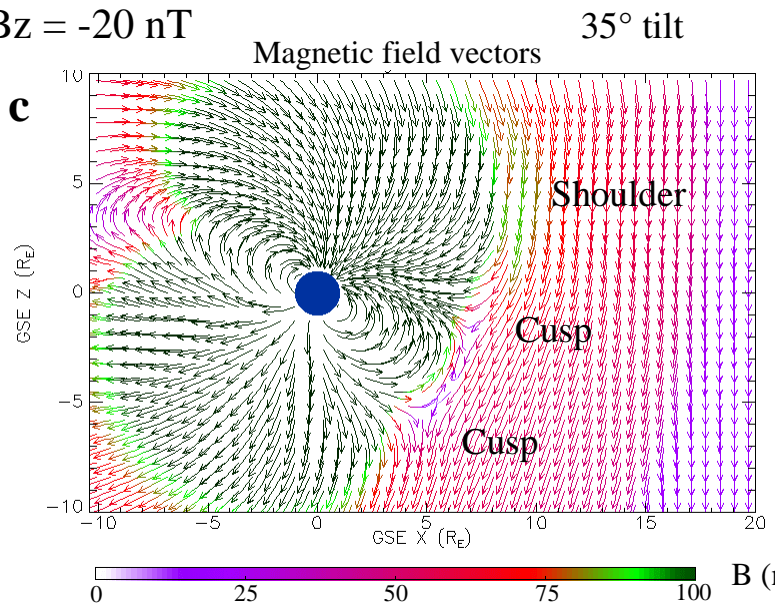

(nT)

Fig. 6. Simulation of the effects of increased dipole tilt and increased magnitude of the IMF. All panels show the $X Z$ plane at $Y=0$. The arrows show the direction of the magnetic field and are colored with the magnitude. (a) and (b) have the same dipole tilt but change the IMF driver. (b) and (c) have the same input driver, but change the dipole tilt. Note that the increased IMF driver compresses the cusps together and starts to build a shoulder of enhanced magnetic field above the cusp that is tilted toward the Sun. Increasing the dipole tilt increases the size and strength of the shoulder.

the magnetopause toward the Earth, away from Polar, and a sharp increase in density was seen at Polar, located at magnetic noon and about $45^{\circ}$ magnetic latitude. This sharp increase was similar to that observed after 09:22 UT on 31 March (Fig. 1) and the two results will be compared in Sect. 7.

Velocity-separator depletion layers are more localized than those found under northward IMF conditions, which extend all around the dayside (Wang et al., 2003). They cannot form if the merging is confined to the subsolar region. Thus, their presence indicates high latitude merging. We reiterate that Polar provides no information on the thickness of a depletion layer. Due to the skimming nature of the trajectory, we lack knowledge about its exact relationship to the magnetopause.

\section{Shoulder depletion layers}

ISM simulations predict a second type of depletion layer with southward $B_{Z}$ when the IMF strength increases. Maynard et al. (2003) gives an example of merging moving away from the equator with increased dipole tilt, with a purely southward IMF. Recall that IMF $B_{X}$ adds or subtracts to the effective dipole tilt (Crooker, 1992). Figure 6 shows the effects of combining dipole tilt and increasing the IMF magnitude. Figure 6 a shows magnetic-field vectors colored with the magnitude of the magnetic field for simulation 2 (Table 1) with $17^{\circ}$ dipole tilt and a $-5 \mathrm{nT} B_{Z}$ as the driver. The magnetopause and cusps are evident in the vector patterns. Increasing the driver to $-20 \mathrm{nT}$ in simulation 3 (Table 1), shown in Fig. $6 \mathrm{~b}$, causes the cusps in the outer magnetosphere to move toward the equator, shrinking the dayside magnetosphere at sub-cusp latitudes. A shoulder, or a region of open field lines, develops above the Northern Hemisphere cusp and extends 


\section{Shoulder Depletion with $\mathrm{Bz}=-\mathbf{2 0} \mathrm{nT}$ and $-\mathbf{3 5}^{\circ}$ dipole tilt}
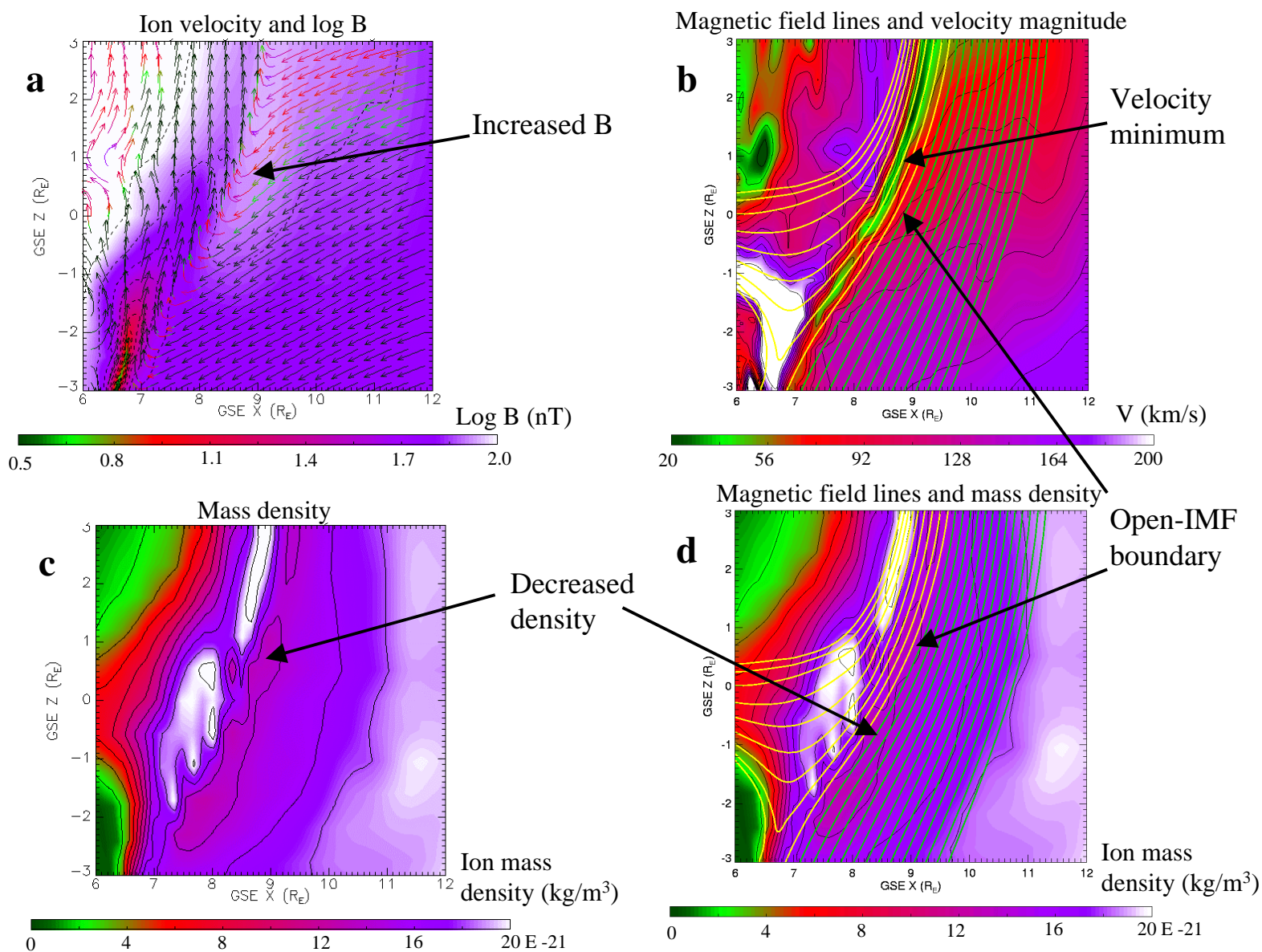

Fig. 7. Details of the shoulder depletion layer depicted in Fig. 6c. All panels display the $X Z$ plane at $Y=0$. (a) Ion velocity vectors overlaid on the plane colored with the $\log$ of $B$. (b) Traced magnetic field lines with the velocity magnitude as the background. The yellow field lines are open and connected to the Northern Hemisphere ionosphere. The green field lines are open to the solar wind on both ends. A velocity minimum is seen just inside the open/IMF boundary. (c) Mass density and (d) mass density with the traced magnetic field line overlay. The decreased density and the enhanced $B$ seen in (a) are co-located at the open-IMF boundary.

sunward of the sub-solar magnetopause. In part, the shoulder results from strong Northern (summer) Hemisphere region 1 currents that close through the low-latitude boundary layer and along the high-latitude magnetopause. Figure $6 \mathrm{c}$ shows the shoulder expanding with increased dipole tilt to $35^{\circ}$. Green colored vectors extending out into the magnetosheath above the cusp indicate the presence of a large magnetic field and the possibility of a depletion layer. The shoulder represents the first point of contact between a purely southward IMF and the magnetosphere. Magnetic field vectors in Figs. $6 \mathrm{~b}$ and $\mathrm{c}$ are seen to drape around the shoulder.

Figure 7 displays plasma densities and magnetic fields observed near the magnetopause poleward of the NorthernHemisphere cusp for simulation 4 (Table 1), depicted in Fig. 6c. Figure 7 a plots velocity vectors over contours of the $\log$ of the magnetic field. Figure $7 \mathrm{~b}$ traces magnetic field lines over contours of the magnitude of ion velocity. Traced field lines are colored yellow if they are open with one foot in the Northern Hemisphere ionosphere and green if both ends connect to the solar wind. The change in color marks the open-IMF field boundary or "outer separatrix" extending to an active merging separator. Because of the dipole tilt merging occurs below the equator in the Southern Hemisphere. Thus, the Northern Hemisphere cusp contacts the magnetosheath south of the equatorial plane. The light purple in the region near $X=9$ and $Z=1-3 R_{E}$ indicates an increase in $B$ in the shoulder region at the outer separatrix. The green region behind the outer separatrix in Fig. $7 \mathrm{~b}$ represents a plasma velocity minimum that occurs in a stagnation region where velocity vectors turn poleward and magnetic field lines are swept back over the magnetopause. Figures $7 \mathrm{c}$ and $\mathrm{d}$ show plasma density contours. Magnetic field line traces are also overlaid in Fig. 7d to highlight the outer separatrix by the change from yellow to green magnetic field lines. The reddish color overlaid by the separatrix reflects decreased plasma density in the depletion layer. The magnetic 


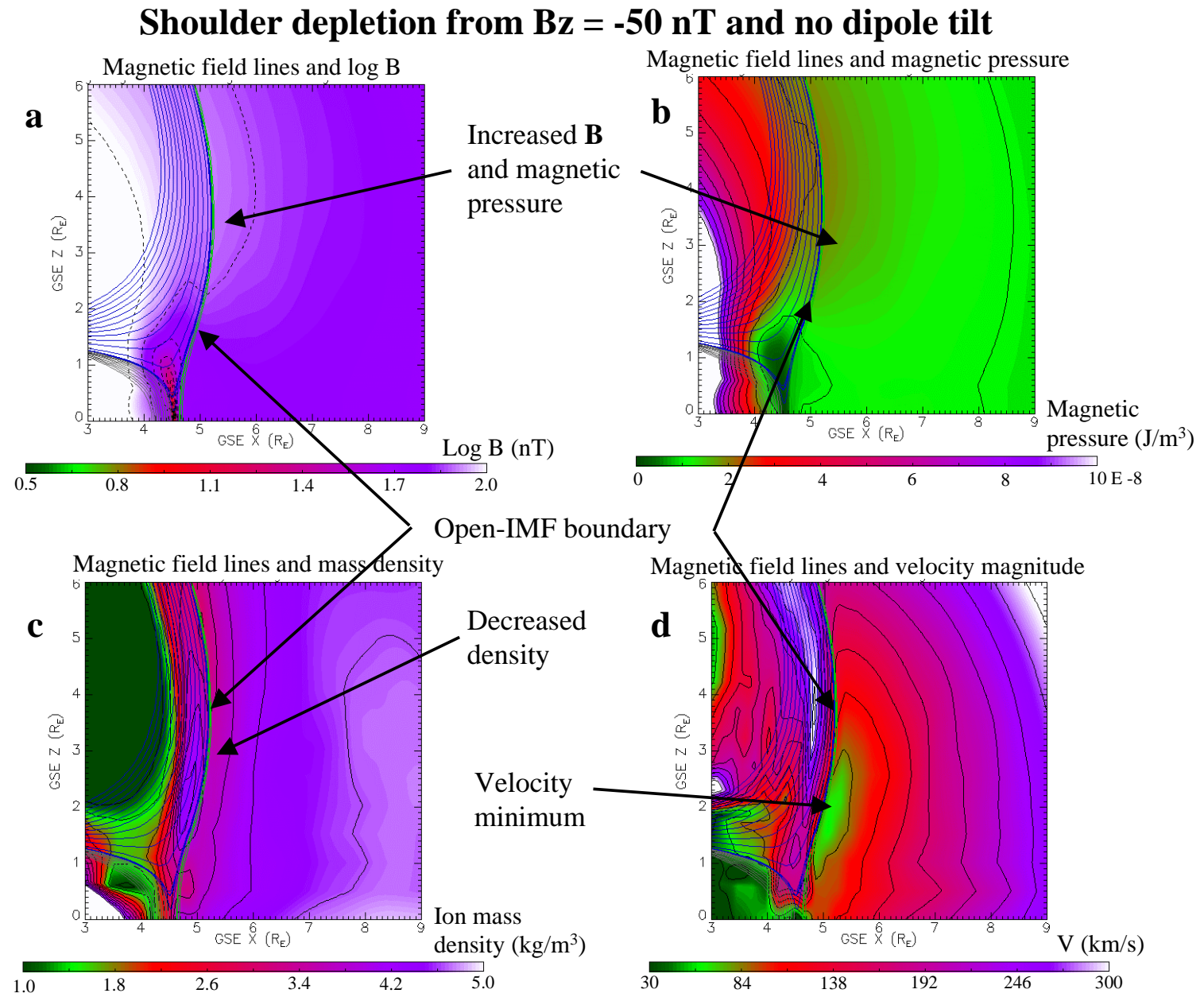

Fig. 8. Shoulders develop above both cusps in simulations with no dipole tilt, but with exceptionally strong driving IMF (50 nT). The panels display a region of the $X Z$ plane at $Y=0$. Magnetic field lines displayed are open and attached to the Northern Hemisphere ionosphere. The backgrounds in (a) and (b) are the log of $B$ and magnetic pressure, respectively. (c) The plane is colored with the mass density. (d) Velocity magnitude provides the background. The depletion layer signatures are highlighted by the arrows, and their location relative to the open-IMF boundary is provided by the traced magnetic field lines.

flux pileup and the decrease in density are clear signatures of a low-shear depletion caused by the shoulder.

If the system is driven even harder, shoulders can develop in both hemispheres with no dipole tilt and a purely southward IMF $B_{Z}$. Figure 8 displays such a case with a driving field of $-50 \mathrm{nT}$ (simulation 5 of Table 1). This run is north-south symmetric and only the Northern Hemisphere is shown. Figures $8 \mathrm{a}$ and $\mathrm{b}$ show the magnetic field and magnetic pressure overlaid with traced magnetic field lines. Blue colored magnetic field lines are open with one foot in the northern ionosphere. Only the first IMF (green) field line is shown, which with the last blue trace defines the outer separatrix, as indicated by an arrow in each plot. Note the increase in magnetic field and in magnetic pressure around the separatrix. Figures $8 \mathrm{c}$ and d show the density and velocity magnitude overlaid with the traced magnetic field lines. Decreased plasma density and velocity are associated with increased magnetic field magnitude. All of these features occur poleward of the cusp and are caused by the shoulder. From symmetry with no dipole tilt, a similar depletion layer develops poleward of the Southern Hemisphere cusp.

The major magnetic storm on 31 March 2001 allowed an empirical test for the existence of shoulder-depletion layers. Figure 9 provides data for 05:00 to 08:00 UT in the same format as Fig. 1. The IMF has a magnitude of $45 \mathrm{nT}$ throughout this period with a clock angle near $160^{\circ}$. At this time, the dipole tilted back toward the nightside in the Northern Hemisphere, emphasizing the shoulder in the vicinity of Polar at southern high latitudes. Note, this is a Southern Hemisphere pass in which Polar transitioned from the mantle into the high altitude cusp and then to the magnetosheath. As evidenced by the continuously negative $B_{Z}$ in Fig. 9a, Polar entered the magnetosheath poleward of the cusp at 06:20 UT, without crossing the shrunken dayside magnetosphere (cf. Figs. 7 and 8). At Polar's prenoon location the magnetic latitude was only $-26^{\circ}$, consistent with the predicted compressed dayside 


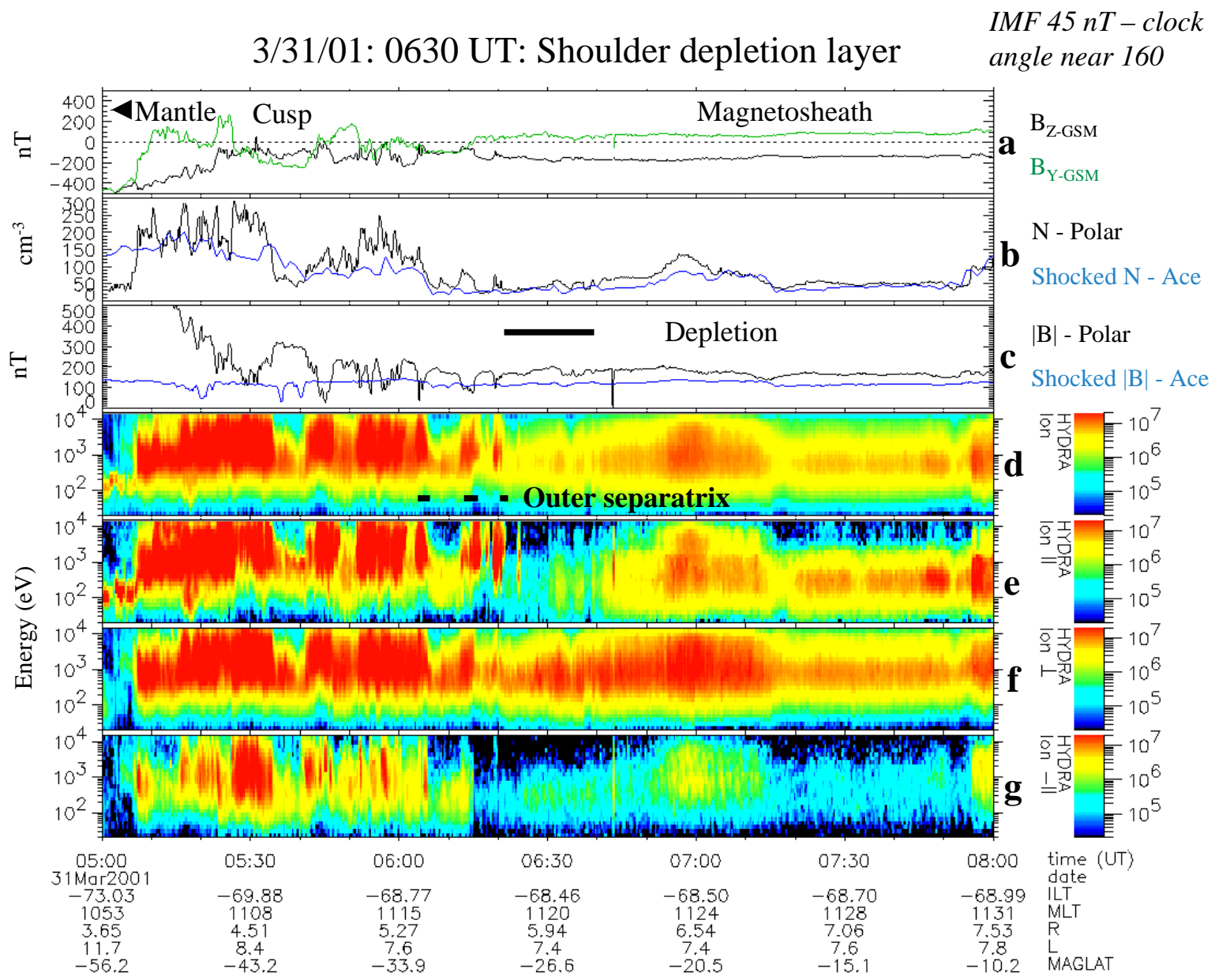

Polar prenoon at $-27^{\circ}$ MLAT and 1120 MLT - never enters dayside magnetosphere

Fig. 9. Polar data from 31 March 2001, showing a shoulder depletion layer located below the Southern Hemisphere cusp in the same format as Fig. 1. Because of the strong driving conditions Polar crossed from the mantle into the magnetosheath, not contacting the dayside magnetosphere. In this case the dipole is tilted away from the Sun.

magnetosphere and equatorward retreat of the cusps. The magnetopause contracted to inside of geosynchronous orbit (Ober et al., 2002). Intense parallel ion fluxes (Fig. 9d) at this time are products of dayside merging causing plasma to stream along the outer separatrix. Ions accelerated parallel to the magnetic field (away from the equator) and similarly directed parallel Poynting flux from Alfvén waves indicate the location of an outer separatrix connected to a merging site at lower latitudes (see black bars in Fig. 9d). The magnitude of $B$ in the magnetosheath at this location above the cusp increased on the magnetosheath side of the separatrix. Accelerated $\mathrm{O}^{+}$ions were detected by the TIMAS instrument on Polar within the cusp and at the separatrix (not shown). The cutoff in $\mathrm{O}^{+}$ion fluxes confirms Polar's transition into the magnetosheath. Low density and lower energy perpendicular fluxes are measured between 06:20 and 06:40 UT. The density is comparable to the lagged ACE density. All of these features indicate the presence of a depletion layer. As the lagged ACE density increased near 07:00 UT, the energy and intensity of magnetosheath ions increased even more. The magnetopause boundary moved farther away from the satellite at that time. Later, as the solar wind density observed by ACE decreased and the magnetopause apparently expanded closer to Polar, ion fluxes measured after 07:15 UT again decreased in energy and intensity.

Later in the day, between 15:00 and 17:00 UT, while geomagnetic activity was still strong, Polar re-entered the magnetosphere poleward of the Northern Hemisphere cusp. The IMF was $30 \mathrm{nT}$ with a clock angle near $180^{\circ}$. At this time the Earth's dipole tilted sunward, emphasizing the formation of a Northern Hemisphere shoulder. Figure 10 presents Polar data in the same format as above. The change from positive to negative $B_{Z}$ near 14:30 UT occurred in the magnetosheath and reflects a change in the imposed IMF direction. At 16:00 UT the satellite was near magnetic noon and $47^{\circ}$ magnetic latitude. The negative direction of $B_{Z}$ is consistent with the direction of the IMF and of open field lines above the Northern Hemisphere cusp. Accelerated antiparallel fluxes 


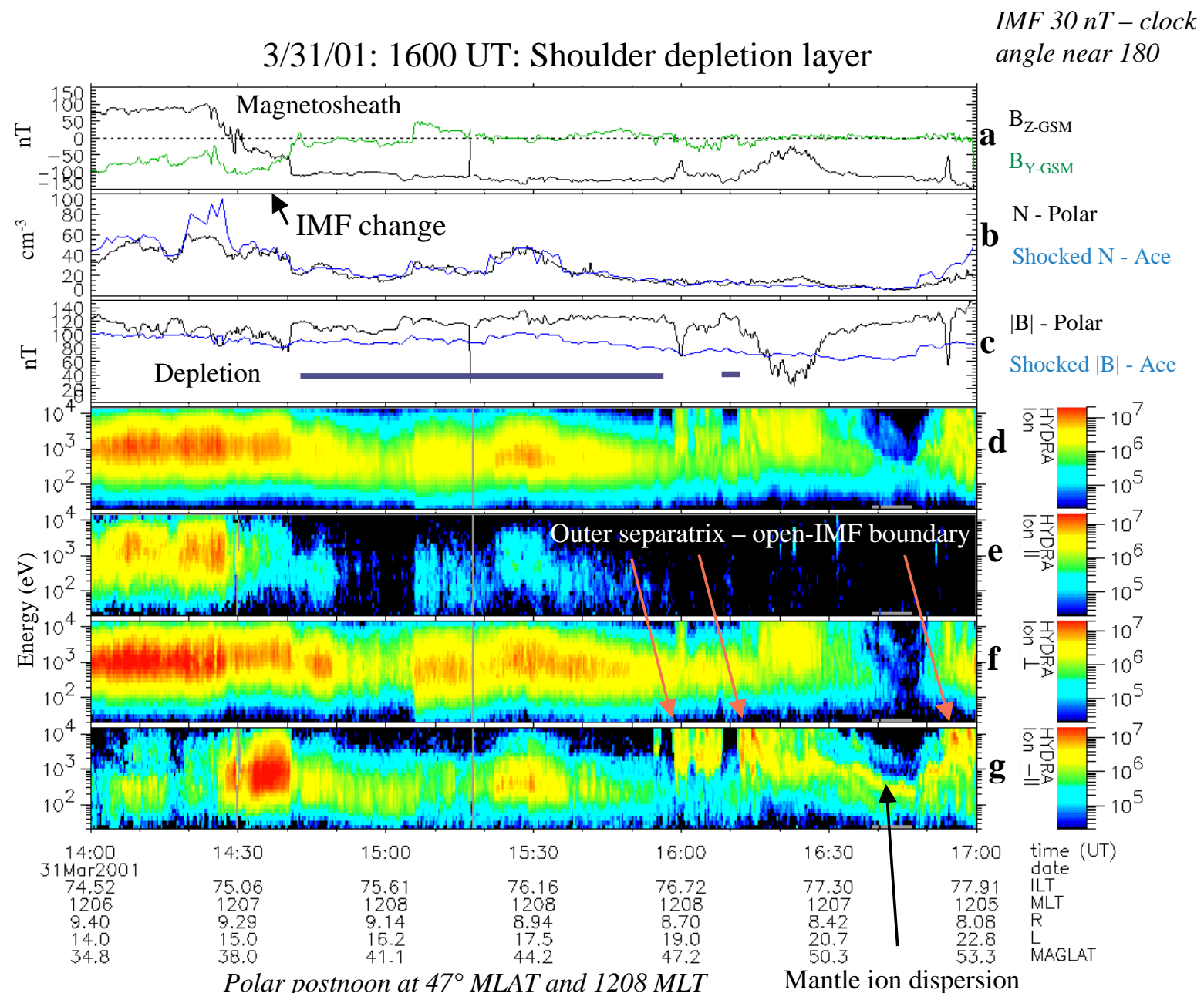

Fig. 10. Polar data from 31 March 2001, inbound above the Northern Hemisphere cusp showing a shoulder depletion layer. The format is the same as in Fig. 1. In this case (later in the day from the data shown in Fig. 9) the dipole was tilted toward the Sun, as Polar crossed from the magnetosheath to the mantle.

of ions and Poynting flux were observed, indicating a crossing of the outer separatrix. Subsequently, the plasma density and magnetic fields were changing, allowing for motion of the magnetopause. Two other outer separatrix crossings are identified in Fig. 10. At each separatrix crossing the magnetic field was larger on the magnetosheath side. Throughout the period when Polar was near the IMF-open boundary, the plasma density was low and comparable to the lagged solar wind density, supporting our interpretation that Polar observed a shoulder depletion layer. Near 16:45 UT the ion fluxes became more characteristic of the mantle. The increase in the ACE density starting at that time recompressed the magnetosphere, returning the separatrix to the location of Polar at 16:53 UT.

These two examples provide evidence for the existence of shoulders and shoulder depletion layers. In each case the density was low in the vicinity of the IMF-open field line boundary as predicted by the simulations.

\section{Depletion layer structure}

Southwood and Kivelson (1995) suggested that density enhancements and depletion layers in front of the magnetopause were complementary parts of a standing slow mode structure. Two of the depletion layer observations discussed above allowed us to test this hypothesis with both northward and southward IMF components.

To make quantitative comparisons with this slow mode concept of depletion layers and its surroundings, we have tested whether the observed density and magnetic field fluctuations are consistent with the proposed type disturbances. Scudder et al. (2003) developed a test for slow-mode characteristics of the observed time domain structures, observed using Galilean-invariant quantities. Specifically, the dispersion relation for slow mode waves predicts that Fourier amplitudes of the density, $\delta \rho$ and magnetic field variations $\delta b_{y}$ should be linearly anti-correlated:

$\delta n /\left.n_{o}\right|_{S}=-\alpha \sin \theta_{B k} \delta b_{y} / B_{o}$. 

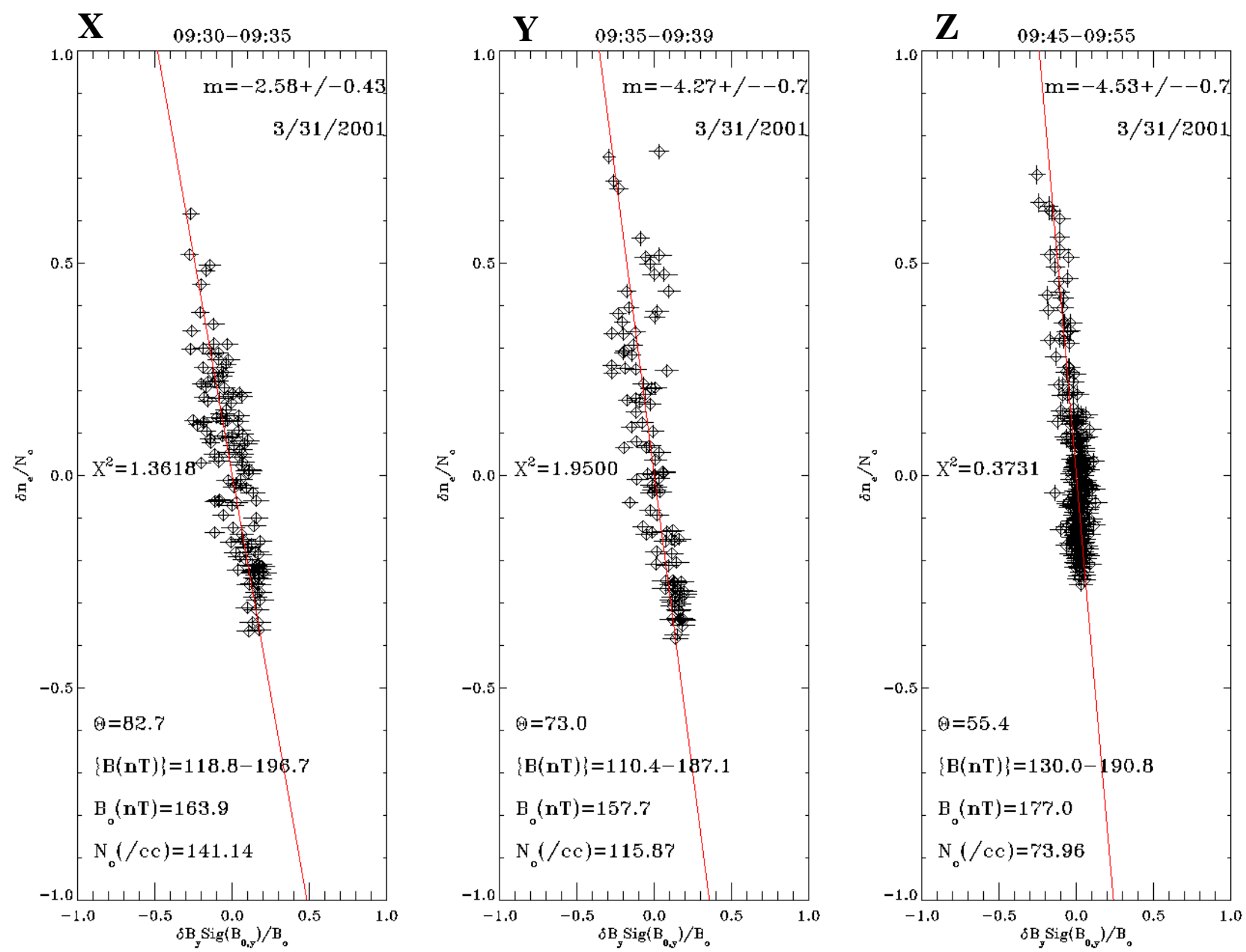

Fig. 11. Linear regression fits for three intervals of 31 March 2001, labeled X, Y, and Z in Fig. 1. Normalized changes in density are plotted as functions of normalized changes in the maximum variance component of $B . B_{O}$ and $N_{O}$ represent values of $B$ and $N$ at the nominal fit zero of the plane wave and are constrained to be within the interval. The actual zero may lie outside the interval. The function Sig(x), -1 for $X<0$ and +1 for $X>0$, is used to rectify the sign of $\delta B$ so that negative values of the resulting slope represent slow-mode solutions. $\operatorname{Sig}(0)=0$. The reported slope $\mathrm{m}$ (related to $\alpha$ in Eqs. (1) and (2)) is $\mathrm{M} / \sin \left(\Theta_{b} k\right.$ ), where $\mathrm{M}$ represents actual slopes obtained from the plotted lines. See Scudder et al. (2003) for more details.

Here, $\alpha>0, \theta_{B K}$ is the propagation angle and $B_{O}$ is the magnetic field intensity for the interval. $n_{o}$ and $P_{o}$ are plasma variables occurring when the mean magnetic field is actually observed.

The usual MHD description of the slow mode assumes polytropic closure to determine a sound speed, $C_{S}$, which is the proportionality constant in the relationship between density and pressure fluctuations: $\delta P=C_{s}^{2} \delta n$. Also, $C_{s}^{2}=\gamma P_{o} / n_{o}$, where $P_{o}$ and $n_{o}$ are the spatially uniform reference pressure and density. The dimensionless proportionality constant in Eq. (1) is determined from linear theory to be

$\alpha=\frac{V_{A}^{2}}{\left(C_{s}^{2}-U^{2}\right)}$,

where $U$ is the phase velocity of the wave in the plasma rest frame. For the slow-mode branch, the denominator of Eq. (2) is positive for all $\beta$ and directions of wave propagation. To test for the slow mode signatures we compare data on the right- and left-hand sides of Eq. (1) to a linear model without intercept in a coordinate system determined by minimum variance analysis on B, as discussed by Scudder et al. (2003).

The ordinate of the fit is

$\delta n(t) \equiv n(t)-n_{o}$

while the abscissa is determined by

$\delta b_{y}(t)=\mathbf{B}(t)-B_{y o} \cdot \hat{\mathbf{y}}$.

A correct inference of $\delta b_{y}$ is contingent on having correctly identified $\hat{\mathbf{y}}, B_{o}, \theta$. We optimize the intercept-free linear regression between $\delta n / n_{o}$ and $\sin \left(\theta_{B k}\right) \delta b_{y} / B_{o}$. If after consideration of the errors, the regression is acceptably ranked, the best-fit slope $\langle\alpha\rangle_{\text {wave }}$ is compared with the theoretical 
prediction of Eq. (1) evaluated at the optimal $\left(B_{o}, \theta_{B k}\right)$. The regression slope, together with $\beta$, implies a correlative choice for $\gamma(\langle\alpha\rangle)$. Plasma data can also be analyzed separately to determine the underlying value of for $\gamma_{\text {plasma }}$ independent of the wave fitting described above. Our final consistency check is to show that $\langle\alpha\rangle_{\text {wave }} \approx\langle\alpha\rangle_{\text {plasma }}$. The more extensive analysis of Scudder et al. (2003) also allows for wave packets in two orthogonal directions to the principal propagation defined by the minimum variance analysis. These additional waves, which may be slow or fast mode waves, are necessary to fully reconstruct the density variation versus time profile. Since they are not necessary for this study, we have omitted them here.

Figure 11 illustrates results of the procedure performed during the three intervals of 31 March 2001, indicated by X, $\mathrm{Y}$, and $\mathrm{Z}$ in Fig. 1. The data and optimal linear fits are given, as well as the slope-fit parameters, uncertainties, normalized $\chi^{2}$, and optimized values of $B_{o}, n_{o}$ and $\theta$, and their uncertainties (note that this fit procedure selects for $B_{o}$ a value that lies within the fit interval). High quality linear fits with negative slopes characteristic of slow mode waves are found by this technique, with similar values of $B_{o}$. The densities decrease with time, as do the adjusted ACE data. The population near the regression lines also provides information about the relative importance during each interval of plasma compression or rarefaction. If both behaviors occur in an interval, data points span a range in $\delta n$ that straddles zero in a significant way. Conversely, in intervals principally characterized as compressions (rarefactions) data points cluster along the fit line primarily in the second (fourth) quadrant of the diagram. Our determinations of $\delta n(t)$ really represent our measure of $\delta n e^{i k z(t)}$ as the wave train is carried past our viewing position. Data points do not represent simple Fourier amplitudes of some mode, but spatially modulated functions, that cause our estimates to be distributed along a line rather than cluster about a point on the line appropriate for the ratio of pure Fourier amplitudes. Our diagnostic is immune to the vagaries of relative motion, fitting quantities that are Galilean invariant: the relative opposed phase of the density and magnetic field strength.

The regression analysis provides strong support for the slow mode playing a significant role in density/field variations observed in all three regions. To reduce ambiguities inherent in the variable densities observed by ACE, our first interval for analysis commenced at 09:30 UT. Negative slopes characteristic of slow-mode structures are evident on both sides of the density peak. In interval $\mathrm{Z}$ the slow mode slope was similar to that of interval $\mathrm{Y}$, with a large percentage of the points located below the plot's centerline. Comparing Fig. 11 with Fig. 1b, we conclude that when the density peak was reached (probably in the compressive phase of the wave), relative motion between the spacecraft and boundary moved Polar back into the region of stronger depletion. This interpretation is consistent with variations in Polar and ACE $\mathbf{B}$ and density data shown in Fig. 1. As the magnetopause breathed outward Polar moved back into the principal depletion region. That the data points fit straight lines so well is a measure of the source's generation of pure slow-mode waves in a nondispersive regime. There is no need for a wave packet be monochromatic as long as its constituent wave numbers lie within the same nondispersive branch. The duration that Polar remained within features suggests that a slow-mode wave is standing in front of the magnetopause, as proposed by Southwood and Kivelson (1995).

Figure 12 show data from the three intervals labeled $X$, $\mathrm{Y}$, and $\mathrm{Z}$ in Fig. 5. While Fig. 11 illustrates a standard depletion with northward IMF, the case shown in Fig. 12 occurred during a relatively high shear event with southward IMF. Panel (X) confirms the presence of the slow mode wave in the interval before 08:00 UT, previously identified as a depletion. Panels (Y) and (Z) show slow-mode behavior on both sides of the large change in density imposed from the solar wind near 10:23 UT. The actual period of the rapid change was omitted. In this case the density peaks above adjusted ACE values. The increased parallel and anti-parallel fluxes suggest that the spacecraft stayed on the outside of the slow-mode enhancement after 10:23 UT. In the subsequent interval the fits (not shown) varied in slope and $\chi^{2}$ increased. It is conceivable that at a given time Polar could simultaneously witness compressive wave power from multiple sources, thus destroying correlations recorded at successive times by Polar. In the 16 April case the second depletion formed while the IMF rotated from negative to positive $B_{Y}$. The density increased when a strong density enhancement, seen by the ACE, encountered the magnetopause. The increased dynamic pressure caused the magnetopause to move inward, allowing Polar to move from the depleted to the enhanced density part of the standing wave. Unlike the 31 March event, there was no imposed density decrease allowing the magnetopause to push back toward the spacecraft. Thus, Polar observed the outer compressive portion of the slow mode wave.

Finally, we note that we found evidence (not shown) for slow-mode waves in the other illustrated depletion layers.

\section{Discussion}

In the previous sections we presented empirical and simulation evidence for the existence of two types of depletion layers forming near the magnetopause when the IMF has a southward component. In both cases Polar observations support the predictions of ISM simulation. While these depletion layers occur under relatively unusual circumstances, their presence in ISM simulations provides clues as to when and where they may exist. In the following we further explore the implications of these findings.

\subsection{Velocity-separator depletion layers}

Maynard et al. (2003) provided evidence for a depletion layer developing while the IMF clock angle was about $140^{\circ}$. This paper confirms that interpretation by showing a region of depleted density relative to ACE measurements near the 

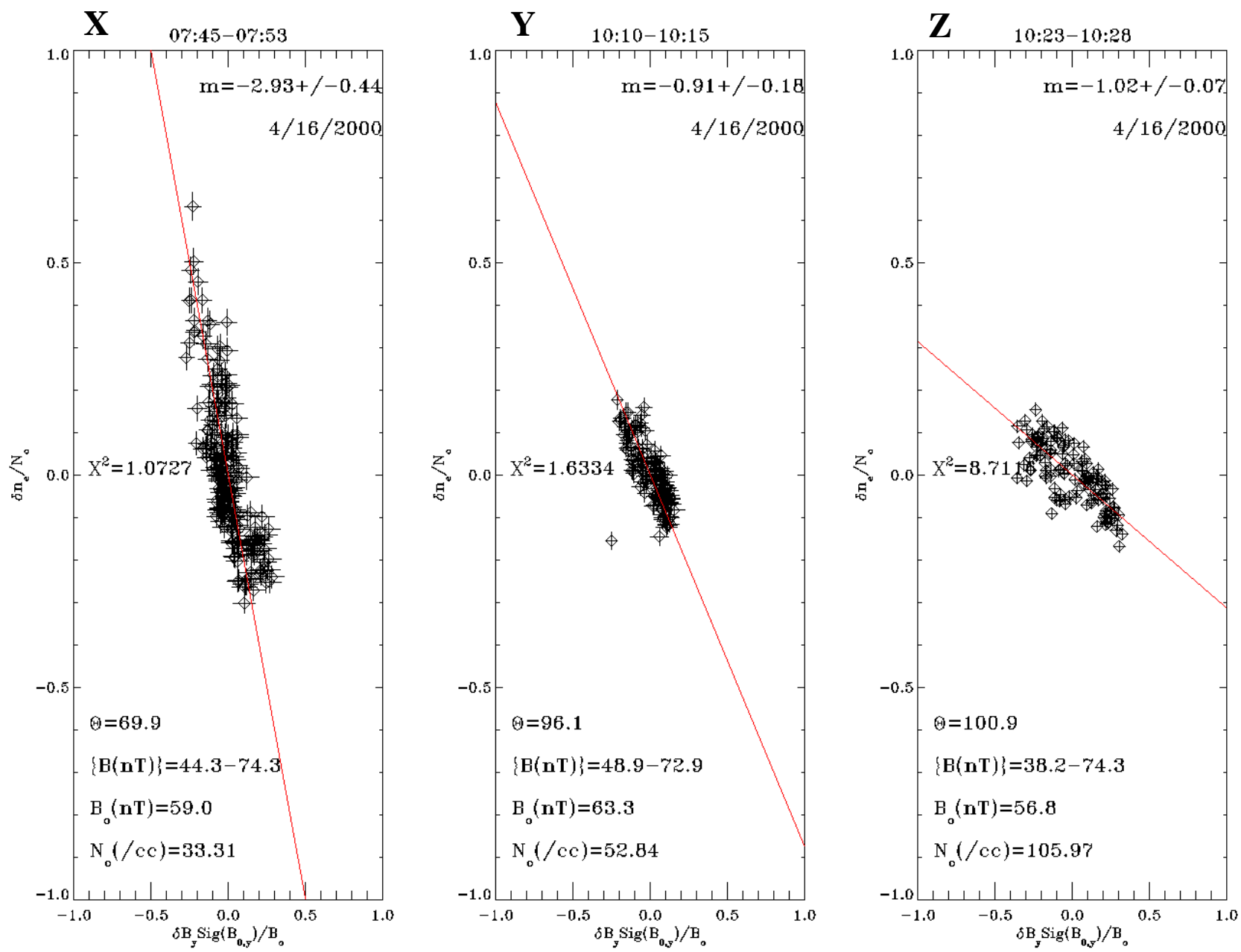

Fig. 12. Linear regression fits for three intervals of 16 April 2000, labeled X, Y, and Z in Fig. 5. See caption for Fig. 11.

location of velocity separator. ISM reveals that the depletion develops best in the presence of a significant $B_{X}$ and/or dipole tilt. Crooker et al. (1992) showed that the effects of $B_{X}$ and dipole tilt are similar and may either add or subtract, depending on polarity. Figure 19 of Maynard et al. (2003) showed that as the dipole tilt increases, the merging site moves to higher latitudes away from the tilt. This lengthens the time for draping of the IMF field lines around the magnetopause to the high-latitude site. We suggest that prolonged draping times allow magnetic flux to pile up and consequent depletion layers to form. Component merging at low latitudes is inconsistent with this flux pileup. The presence of the depletion layer supports our interpretation of observations presented in Maynard et al. (2003), that magnetic merging was proceeding at high latitudes poleward of the spacecraft. In any situation in which the merging site is remote and additional draping time is involved to reach that merging site, we may expect depletion to occur.

\subsection{Shoulder depletion layers}

We have developed evidence for the existence of shoulder depletion layers in the Polar data and ISM simulations. Two effects occur: the dayside cusp at the magnetopause is pinched toward the equator and a shoulder develops above the cusp. Both effects can be explained as fringe-field effects from Region 1 currents closing through the high-latitude ionosphere over the polar cap. Siscoe et al. $(1991,2002 c)$ show that associated current loops pass through the magnetopause above the cusp, which augment/modify the Chapman-Ferraro currents in that region. These currents form a loop where the field internal to the loop has strong $+X$ and $-Z$ components, which adds to the magnetic field above the cusp and subtracts below the cusp. This perturbation magnetic field contributes to shoulder development. Similarly, it depresses the magnetic field in the sub-solar region. The magnetic field at the nose drops below the dipole field for IEF values of $4 \mathrm{mV} \mathrm{m}^{-1}$. 
Sunward dipole tilts push the shoulder region forward. This increases the time required for the IMF to drape to the merging site and allows density depletions to deepen. Even at equinox the dipole tilt varies $\pm 12^{\circ}$ every day, and the tilt is strongest at solstice. IMF $B_{X}$ causes complementary variations (Crooker et al., 1992). The IEF phase plane is generally tilted, with large $B_{X}$ increasing the tilt in the $X Z$ plane, so that the phase plane tilt may add or subtract from the dipole tilt to provide an effective tilt that in some cases is much greater. The IEF phase plane orientation varies on time scales of $10 \mathrm{~s}$ of min (Weimer et al., 2002). Thus, the depths of depletions may be dynamic quantities, depending on the values of all three IMF components and the dipole tilt.

\subsection{Slow mode structure of depletion layers}

We have examined Polar measurements for evidence of slow mode waves standing in front of the depletion layer as postulated by Southwood and Kivelson (1995). In fact, evidence for a density enhancement and low $B$ appears near 12:30 UT in Fig. 2. However, it was shown in Maynard et al. (2003) that this slow-mode-like feature reflected similar variations in the solar wind and was not unique to the formation of a depletion layer. Figure $2 \mathrm{c}$ shows that magnetic fields observed at Polar track shocked values of ACE measurements quite well, confirming that the structure was imposed by the solar wind. Because of the skimming nature of its orbit, Polar did not fully penetrate to magnetosheath features standing in front of the magnetopause, unless there were variations in the drivers. Variations in dynamic pressure change the position of the magnetopause and the relative position of Polar within the layer itself. In two of the cases described above, the variations were sufficient for Polar to see both the density minimum and at least some of the density enhancement portions of standing slow-mode waves. The long duration spent by Polar within depletion layers is the basis for our contention that the structure is a wave standing in front of the magnetopause.

Just as the nose of the magnetopause presents an obstacle around which the IMF must drape, causing the depletion layer to form, so do the Region 1 current loops and their shoulders poleward of the cusp. Because shoulders inhibit inward flow until draping allows merging to occur, a depletion layer characterized by increased $B$ and decrease density must form. The Southwood and Kivelson (1995) scenario would require a slow mode shock to form and stand off the magnetopause, which would turn the field. Next to the magnetopause a rotation in $B$ is required and the depletion layer is formed. This layer, which occurs above the cusp, is a lowshear depletion.

\section{Summary}

We have provide observational and MHD simulation evidence for the existence of depletion layers in specific regions and situations when the IMF has southward components.
We have furnished observational evidence in support of the Southwood and Kivelson (1995) hypothesis that density depletions (Zwan and Wolf, 1976) and enhancements (Song et al., 1990; 1992) are parts of a slow mode wave standing in front of the magnetopause. The existence of depletion layers in front of a dayside, high-shear magnetopause with southward IMF components requires that any merging must be remote from the observation. Their presence in the sub-solar region favors high latitude merging and rules out sub-solar component merging, if merging occurs on that field line.

Acknowledgements. We thank F. S. Mozer, C. T. Russell, and W. K. Peterson for Polar electric field, magnetic field, and ion composition data, and C. W. Smith and D. J. McComas for ACE magnetic field and plasma data. We especially thank M. Kivelson for helpful discussions. This work was supported in part by AFOSR Task 2311SDA3, NASA grants NAG5-8135 (Sun-Earth Connections Theory Program grant) and NASW-99014, and by the NASA Polar mission through subcontracts with the University of California at Berkeley. The work at Iowa is supported by NASW5-7883 by the Polar Project office at NASA. The ISM was developed under sponsorship of the Defense Threat Reduction Agency, 45045 Aviation Drive, Dulles, VA 20166-7517.

Topical Editor T. Pulkkinen thanks a referee for his help in evaluating this paper.

\section{References}

Anderson, B. J. and Fusilier, S. A.: Magnetic pulsations from 0.1 to $4.0 \mathrm{~Hz}$ and associated plasma properties in the Earth's subsolar magnetosheath and plasma depletion layer, J. Geophys. Res., 98, 1461, 1993.

Anderson, B. J., Phan, T.-D., and Fuselier, S. A.: Relationships between plasma depletion and subsolar reconnection, J. Geophys. Res., 102, 9531-9542, 1997.

Crooker, N. U.: Dayside merging and cusp geometry, J. Geophys. Res., 84, 951-959, 1979.

Crooker, N. U.: Reverse convection, J. Geophys. Res., 97, 1936319372, 1992.

Crooker, N. U., Eastman, T. E., and Stiles, G. S.: Observations of plasma depletion in the magnetosheath at the dayside magnetopause, J. Geophys. Res., 84, 869-874, 1979.

Dungey, J. W.: Interplanetary magnetic field and the auroral zones, Phys. Rev. Lett., 6, 47, 1961.

Farrugia, C. J., Erkaev, N. V., Biernat, H. K., and Burlaga, L. F.: Anomalous magnetosheath properties during Earth passage of an interplanetary magnetic cloud, J. Geophys. Res., 100, 19245 , 1995.

Hain, K.: The partial donor cell method, J. Comp. Physics, 73, 131, 1987.

Harvey, P., Mozer, F. S., Pankow, D., Wygant, J., Maynard, N. C., Singer, H., Sullivan, W., Anderson, P. B., Pfaff, R., Aggson, T., Pedersen, A., Fälthammar, C.-G., and Tanskannen, P.: The electric field instrument on the Polar satellite, Space Sci. Rev., 71, 583-596, 1995.

Lyon, J. G.: MHD simulations of the magnetosheath, Adv. Space Res., 14, (7), 21-28, 1994.

Maynard, N. C., Ober, D. M., Burke, W. J., Scudder, J. D., Lester, M., Dunlop, M., Wild, J. A., Grocott, A., Farrugia, C. J., Lund, E. J., Russell, C. T., Weimer, D. R., Siebert, K. D., Balogh, A., 
Andre, M., and Rème, H.: Polar, Cluster and SuperDARN evidence for high latitude merging during southward IMF: temporal/spatial evolution, Ann. Geophys., 21, 2233-2258, 2003.

McComas, D. J., Bame, S. J., Barber, P., Fieldman, W. C., Phillips, J. L., and Riley, P.: Solar wind electron, proton, and alpha monitor (SWEPAM) on the Advanced Composition Explorer, Space Sci. Rev., 86, 563-612, 1998.

Midgley, J. E. and Davis, L.: Calculation by moment technique of the perturbation of the geomagnetic field by the solar wind, J. Geophys. Res., 68, 5111-5123, 1963.

Ober, D. M., Thomsen, M. F., and Maynard, N. C.: Observations of the bow shock and magnetopause crossings from geosynchronous orbit on March 31, 2001, J. Geophys. Res., 107, (A8), doi:10.1029/2001JA000284, 2002.

Phan, T.-D., Paschmann, G., Baumjohann, W., Sckopke, N., and Lühr, H.: The magnetosheath region adjacent to the dayside magnetopause: AMPTE/IRM observations, J. Geophys. Res., 99, 121-141, 1994.

Raeder, J., Wang, Y. L., Fuller-Rowell, T. J., and Singer, H. J.: Global simulations of magnetospheric space weather effects of the Bastille Day storm, Solar Physics, 204, 325-335, 2001.

Russell, C. T., Snare, R. C., Means, J. D., Pierce, D., Dearborn, D., Larson, M., Barr, G., and Le, G.: The GGS/polar magnetic field investigation, Space Sci. Rev., 71, 563-582, 1995.

Scudder, J. D., Hunsacker, F., Miller, G., Lobell, J., Zawistowski, T., Ogilvie, K., Keller, J., Chornay, D., Herrero, F., Fitzenreiter, R., Fairfield, D., Needell, J., Bodet, D., Googins, J., Kletzing, C., Torbert, R., Vandiver, J., Bentley, R., Fillius, W., McIlwain, C., Whipple, E., and Korth, A.: Hydra: A three dimensional electron and ion instrument for the polar spacecraft of the GGS mission, Space Sci. Rev., 71, 459-495, 1995.

Scudder, J. D., Maynard, N. C., and Burke, W. J.: Slow, fast and mixed compressible modes near the magnetopause, Eos. Trans. AGU, 84, (46), Fall Meet. Suppl., Abstract SM12A-1190, F1254, 2003.

Siscoe, S. L., Lotko, W., and Sonnerup, B. U. Ö.: A high-latitude, low-latitude boundary layer model of the convection current system, J. Geophys. Res., 96, 3487-3496, 1991.

Siscoe, G. L., Crooker, N. U., Erickson, G. M., Sonnerup, B. U. Ö., Siebert, K. D., Weimer, D. R., White, W. W., and Maynard, N. C.: Global geometry of magnetospheric currents inferred from MHD simulations, in Magnetospheric Current Systems, Geophysical Monograph 118, pp. 41-52, Am. Geophys. Union, Washington D.C., 2000.

Siscoe, G. L., Erickson, G. M., Sonnerup, B. U. Ö., Maynard, N. C., Schoendorf, J. A., Siebert, K. D., Weimer, D. R., White, W. W., and Wilson, G. R.: MHD properties of magnetosheath flow, Planet. Space Sci., 50, 461-471, 2002a.

Siscoe, G. L., Erickson, G. M., Sonnerup, B. U. Ö., Maynard, N. C., Schoendorf, J. A., Siebert, K. D., Weimer, D. R., White, W. W., and Wilson, G. R.: Hill model of transpolar potential saturation: Comparisons with MHD simulations, J. Geophys. Res., 107, (A6), doi:10.1029/2001JA000109, 2002b.

Siscoe, G. L., Crooker, N. U., and Siebert, K. D.: Transpolar potential saturation: Roles of region 1 current system and solar wind ram pressure, J. Geophys. Res., 107, (A10), 1321, doi:10.1029/2001JA009176, 2002c.
Smith, C. W., Acuña, M. H., Burlaga, L. F., L’Heureux, J., Ness, N. F., and Scheifele, J.: The ACE Magnetic Field Experiment, Space Sci. Reviews, 86, 613, 1998.

Song, P., Russell, C. T., Gosling, J. T., Thomsen, M., and Elphic, R. C.: Observations of the density profile in the magnetosheath near the stagnation streamline, Geophys. Res. Lett., 17, 2035, 1990.

Song, P., Russell, C. T., and Thomsen, M. F.: Slow mode transition in the frontside magnetosheath, J. Geophys. Res., 97, 82958305, 1992.

Southwood, D. J. and Kivelson, M. G.: On the form of the flow in the magnetosheath, J. Geophys. Res., 97, 2873, 1992.

Southwood, D. J. and Kivelson, M. G.: Magnetosheath flow near the subsolar magnetopause: Zwan-Wolf and SouthwoodKivelson theories reconciled, Geophys. Res. Lett., 22, 3275, 1995.

Sonnerup, B. U. Ö.: The reconnecting magnetosphere, in: Magnetospheric Physics, edited by McCormac, B. M., D. Reidel Publ. Co., Dordrecht, Holland, 23-33, 1974.

Spreiter, J. R., Alksne, A. Y., and Summers, A. L.: External aerodynamics of the magnetosphere, in: Physics of the Magnetosphere, edited by Carovillano, R. L., McClay, J. F., and Radoski, H. R., D. Reidel Publishing Co., Dordrecht, Holland, 301-375, 1968.

Spreiter, J. R. and Alksne, A. Y.: Plasma flow around the magnetosphere, Rev. Geophys., 7, 11-50, 1969.

Spreiter, J. R. and Stahara, S. S.: Magnetohydrodynamics and gasdynamic theories for planetary bow waves, in: Collisionless Shocks in the Heliosphere: Reviews of Current Research, edited by Tsuritani, B. T. and Stone, R. G., Geophysical Monograph 35, AGU, 85-108, 1985.

Wang, Y. L., Raeder, J., Russell, C. T., Phan, T.-D., and Manapat, M.: Plasma depletion layer: Event studies with a global model, J. Geophys. Res., 108, (A1), 1010, doi:10.1029/2002JA009281, 2003.

Whang, Y. C.: Slow Shocks and their Transition to Fast Shocks in the Inner Solar Wind, J. Geophys. Res., 92, 4349, 1987.

Weimer, D. R., Ober, D., Maynard, N. C., Burke, W. J., Collier, M. R., McComas, D. J., Ness, N. F., and Smith, C. W.: Variable time delays in the propagation of the interplanetary magnetic field, J. Geophys. Res., 107, (A8), doi:10.1029/2001JA009102, 2002.

White, W. W., Schoendorf, J. A., Siebert, K. D., Maynard, N. C., Weimer, D. R., Wilson, G. L., Sonnerup, B. U. Ö., Siscoe, G. L., and Erickson, G. M.: MHD simulation of magnetospheric transport at the mesoscale, in: Space Weather, Geophysical Monograph Series, vol. 125, edited by Song, P., Singer, H. J., and Siscoe, G. L., AGU, 229-240, 2001.

Wu, C. C.: MHD flow past an obstacle: Large-scale flow in the magnetosheath, Geophys. Res. Lett., 19, 87-90, 1992.

Zwan, B. J. and Wolf, R. A.: Depletion of solar wind plasma near a planetary boundary, J. Geophys. Res., 82, 1636-1648, 1976. 